\title{
Characterization theorem for classical orthogonal polynomials on non-uniform lattices: The functional approach.
}

\author{
M. Foupouagnigni ${ }^{\diamond}$, M. Kenfack Nangho ${ }^{\diamond}$ and S. Mboutngam ${ }^{\diamond}$ \\ ${ }^{\diamond}$ Department of Mathematics. Higher Teachers Training College \\ University of Yaounde I, Cameroon
}

\begin{abstract}
Using the functional approach, we state and prove a characterization theorem for classical orthogonal polynomials on non-uniform lattices (quadratic lattices of a discrete or a $q$-discrete variable) including the Askey-Wilson polynomials. This theorem proves the equivalence between seven characterization properties, namely the Pearson equation for the linear functional, the second-order divided-difference equation, the orthogonality of the derivatives, the Rodrigues formula, two types of structure relations, and the Riccati equation for the formal Stieltjes function.
\end{abstract}

Keywords: Classical orthogonal polynomials, Non-uniform lattices, Linear functionals, Divided-difference equations, Riccati equation, Structure relations, Functional approach

Mathematics Subject Classification (2010) 33C45, 33D45

\section{Introduction}

Classical orthogonal polynomials (in short COP) on a non-uniform lattice are defined as polynomials $\left(P_{n}\right)_{n}$ with degree $P_{n}=n$, satisfying one of the following orthogonality relations $[4,5,8,13,24]$

$$
\begin{aligned}
& \int_{C} P_{n}(x(s)) P_{m}(x(s)) \rho(s) \nabla x_{1}(s) d s=k_{n} \delta_{n, m}, k_{n} \neq 0, \forall n, m \in \mathbb{N} ; \\
& \sum_{i=0}^{N} P_{n}\left(x\left(s_{i}\right)\right) P_{m}\left(x\left(s_{i}\right)\right) \rho\left(s_{i}\right) \nabla x_{1}\left(s_{i}\right)=k_{n} \delta_{n, m}, k_{n} \neq 0, \forall n, m \in \mathbb{N}, N \in \mathbb{N} \cup\{\infty\},
\end{aligned}
$$

where $\mathbb{N}$ is the set of nonnegative integers, $s_{0}=a, s_{N}=b$. Here, $C$ is an appropriate contour in the complex $s$-plane, and the weight $\rho$ is a solution of the Pearson-type equation

$$
\frac{\Delta}{\nabla x_{1}(s)}(\sigma(s) \rho(s))=\psi(x(s)) \rho(s),
$$

where $\psi$ is a first-degree polynomial and

$$
\phi(x(s))=\sigma(s)+\frac{1}{2} \psi(x(s)) \nabla x_{1}(s)
$$

is a polynomial of degree at most two in $x(s)$, with the border conditions

$$
\left\{\begin{array}{l}
\int_{C} \Delta\left[\sigma(s) \rho(s) x^{k}\left(s-\frac{1}{2}\right)\right] d s=0, k=0,1,2, \ldots \\
\left.\sigma(s) \rho(s) x^{k}\left(s-\frac{1}{2}\right)\right|_{s=a, b}=0, k=0,1,2, \ldots,
\end{array}\right.
$$

\footnotetext{
${ }^{*}$ Corresponding author. Email: foupouagnigni@gmail.com This work was supported by the Alexander von Humboldt Foundation (Bonn, Germany) under the Research Group Linkage Programme 2009-2012, between the Universities of Kassel and Yaounde I
} 
for orthogonality relation (1) and (2) respectively. $\Delta$ and $\nabla$ are the forward and the backward operators

$$
\Delta f(x(s)):=\Delta f(s)=f(s+1)-f(s), \nabla f(x(s)):=\nabla f(s)=f(s)-f(s-1),
$$

and

$$
x_{\mu}(s)=x\left(s+\frac{\mu}{2}\right), \mu \in \mathbb{C},
$$

where $x(s)$ is a non-uniform lattice satisfying $[4,25]$

$$
\begin{aligned}
& x(s+k)-x(s)=\gamma_{k} \nabla x_{k+1}(s), k \geq 0, \\
& \frac{x(s+k)+x(s)}{2}=\alpha_{k} x_{k}(s)+\beta_{k}, k \geq 0,
\end{aligned}
$$

with the sequences $\left(\alpha_{k}\right),\left(\beta_{k}\right),\left(\gamma_{k}\right)$ satisfying the following relations

$$
\begin{aligned}
\alpha_{k+1}-2 \alpha \alpha_{k}+\alpha_{k-1} & =0 \\
\beta_{k+1}-2 \beta_{k}+\beta_{k-1} & =2 \beta \alpha_{k}, \\
\gamma_{k+1}-\gamma_{k-1} & =2 \alpha_{k},
\end{aligned}
$$

and the initial conditions

$$
\alpha_{0}=1, \alpha_{1}=\alpha, \beta_{0}=0, \beta_{1}=\beta, \gamma_{0}=0, \gamma_{1}=1 .
$$

The lattice $x(s)$ is explicitly given by [25]

$$
x(s)= \begin{cases}c_{1} q^{-s}+c_{2} q^{s}+c_{3} & \text { for } \quad \alpha=\frac{q^{\frac{1}{2}}+q^{-\frac{1}{2}}}{2}, \\ c_{4} s^{2}+c_{5} s+c_{6} & \text { for } \quad \alpha=1 .\end{cases}
$$

Costas-Santos and Marcellán [5], using the Pearson equation (3) for the weight function, gave a characterization theorem for classical orthogonal polynomials on the lattice (10), proving the equivalence between:

1. the second-order divided-difference equation

$$
\left\{\sigma(s) \frac{\Delta}{\nabla x_{1}(s)} \frac{\nabla}{\nabla x(s)}+\psi(x(s)) \frac{\Delta}{\Delta x(s)}+\lambda_{n}\right\} P_{n}(x(s))=0, n \geq 0
$$

2. the orthogonality of the derivatives $\left(\frac{\Delta P_{n+1}(x(s))}{\Delta x(s)}\right)_{n}$;

3. the Rodrigues formula

$$
P_{n}(x(s))=\frac{B_{n}}{\rho(s)} \frac{\nabla}{\nabla x_{1}(s)} \cdots \frac{\nabla}{\nabla x_{n}(s)}\left(\rho_{n}(s)\right), \text { with } \rho_{k}(s)=\sigma(s+1) \rho_{k-1}(s+1), \rho_{0}(s):=\rho(s) ;
$$

4. and the second structure relation

$$
\frac{P_{n}(x(s+1))+P_{n}(x(s))}{2}=C_{n, n+1} \frac{\Delta}{\Delta x(s)} P_{n+1}(x(s))+C_{n, n} \frac{\Delta}{\Delta x(s)} P_{n}(x(s))+C_{n, n-1} \frac{\Delta}{\Delta x(s)} P_{n-1}(x(s)),
$$

with $C_{n, n-1} \neq 0$. 
Koornwinder [14] in 2007 gave a structure relation for classical orthogonal polynomials of the form

$$
\mathbb{L}\left(p_{n}\right)(x)=\gamma_{n} A_{n} p_{n+1}(x)-\gamma_{n-1} C_{n} p_{n-1}(x),
$$

where $A_{n}$ and $B_{n}$ are the coefficients of the three-term recurrence relation

$$
x p_{n}(x)=A_{n} p_{n+1}(x)+B_{n} p_{n}(x)+C_{n} p_{n-1}(x),
$$

while $\mathbb{L}$ is a linear operator acting on the linear space $\mathbb{R}[x]$ of polynomials of the variable $x$ with real coefficients. In addition $\mathbb{L}$ is skew symmetric

$$
\langle\mathbb{L} f, g\rangle=-\langle f, \mathbb{L} g\rangle, \forall f, g \in \mathbb{R}[x],
$$

and satisfies

$$
\mathbb{L}\left(x^{n}\right)=\gamma_{n} x^{n+1}+\text { terms of lower degree, }
$$

where $\langle.,$.$\rangle is the inner product with respect to which the corresponding polynomial sequence is orthogonal.$

For the specific case of Askey-Wilson polynomials, Koornwinder gave the operator $\mathbb{L}$ as

$$
(\mathbb{L}(f))[z]=\frac{(1-a z)(1-b z)(1-c z)(1-d z) z^{-2} f[q z]-\left(1-\frac{a}{z}\right)\left(1-\frac{b}{z}\right)\left(1-\frac{c}{z}\right)\left(1-\frac{d}{z}\right) z^{2} f\left[\frac{z}{q}\right]}{z-z^{-1}},
$$

with the notation $f[z]:=f\left(\frac{z+z^{-1}}{2}\right)=f(x)$, where $x=\frac{z+z^{-1}}{2}$. More details are given in Section 4 .

The aim of this paper is to:

1. state the Pearson-type equation for the linear functional of the corresponding classical orthogonal polynomials, and prove that the Pearson equation for the weight implies the one of the linear functional;

2. state and prove using the functional approach seven equivalent characterization properties for classical orthogonal polynomials: the four properties given by Costas-Santos and Marcellán [5], plus, the Pearson equation for the linear functional, the Rodrigues formula for the linear functional, the first structure relation and the Riccati equation for the formal Stieltjes function;

3. find the link between the structure relation given above by Koornwinder [14] and our second structure relation;

4. connect this work with the pioneering one by Magnus [15] who gave the Riccati equation for the associate Askey-Wilson polynomials.

Since the operator $\mathbb{D}_{x}$ reduces to the forward operator $\Delta$ and the Hahn operator $D_{q}\left(D_{q} f(s)=\frac{f(q s)-f(s)}{(q-1) s}\right)$ for the lattices $x(s)=s$ and $x(s)=q^{s}$ respectively [8], this work generalizes previous ones characterizing classical orthogonal polynomials by means of the above mentioned seven equivalent properties. Among these, we would like to mention [1, 2, 17] for COP of a continuous variable, [12] for COP of a discrete variable, $[21,20,3]$ for COP of a $q$-discrete variable and $[5,14]$ for COP on a non-uniform lattice.

In Section 2, we recall known results and link the Pearson equation for the weight with the one of the linear functional. Section 3 deals with the characterization theorem while the last section provides some important connections and perspectives. 


\section{Known Results and Pearson-type Equations}

\subsection{Properties of the Companion Operators $\mathbb{D}_{x}$ and $\mathbb{S}_{x}$}

By means of the companion operators $\mathbb{D}_{x}$ and $\mathbb{S}_{x}[8]$

$$
\mathbb{D}_{x} f(x(s))=\frac{f\left(x\left(s+\frac{1}{2}\right)\right)-f\left(x\left(s-\frac{1}{2}\right)\right)}{x\left(s+\frac{1}{2}\right)-x\left(s-\frac{1}{2}\right)}, \mathbb{S}_{x} f(x(s))=\frac{f\left(x\left(s+\frac{1}{2}\right)\right)+f\left(x\left(s-\frac{1}{2}\right)\right)}{2},
$$

Equation (11) can be rewritten as $[7,8]$

$$
\phi(x(s)) \mathbb{D}_{x}^{2} P_{n}(x(s))+\psi(x(s)) \mathbb{S}_{x} \mathbb{D}_{x} P_{n}(x(s))+\lambda_{n} P_{n}(x(s))=0,
$$

where

$$
\lambda_{n}=-\gamma_{n}\left(\phi_{2} \gamma_{n-1}+\psi_{1} \alpha_{n-1}\right) .
$$

The operators $\mathbb{D}_{x}$ and $\mathbb{S}_{x}$, which transform a polynomial of degree $n$ in the variable $x(s)$ into a polynomial of degree $n-1$ and $n$ respectively in $x(s)$, fulfill important relations-which read, taking into account the shift (compared to the definition in [8]) in the definition of the above defined companion operators, as

Theorem 1 [8]

1. The operators $\mathbb{D}_{x}$ and $\mathbb{S}_{x}$ satisfy the product rules $I$

$$
\begin{aligned}
& \mathbb{D}_{x}(f(x(s)) g(x(s)))=\mathbb{S}_{x} f(x(s)) \mathbb{D}_{x} g(x(s))+\mathbb{D}_{x} f(x(s)) \mathbb{S}_{x} g(x(s)), \\
& \mathbb{S}_{x}(f(x(s)) g(x(s)))=U_{2}(x(s)) \mathbb{D}_{x} f(x(s)) \mathbb{D}_{x} g(x(s))+\mathbb{S}_{x} f(x(s)) \mathbb{S}_{x} g(x(s)),
\end{aligned}
$$

where $U_{2}$ is a polynomial of degree 2

$$
U_{2}(x(s))=\left(\alpha^{2}-1\right) x^{2}(s)+2 \beta(\alpha+1) x(s)+\delta_{x},
$$

and $\delta_{x}$ is a constant depending on $\alpha, \beta$ and the initial values $x(0)$ and $x(1)$ of $x(s)$ :

$$
\delta_{x}=\frac{x^{2}(0)+x^{2}(1)}{4 \alpha^{2}}-\frac{\left(2 \alpha^{2}-1\right)}{2 \alpha^{2}} x(0) x(1)-\frac{\beta(\alpha+1)}{\alpha^{2}}(x(0)+x(1))+\frac{\beta^{2}(\alpha+1)^{2}}{\alpha^{2}} .
$$

2. The operators $\mathbb{D}_{x}$ and $\mathbb{S}_{x}$ satisfy the quotient rules

$$
\begin{aligned}
\mathbb{D}_{x}\left(\frac{f(x(s))}{g(x(s))}\right) & =\frac{\mathbb{S}_{x} f(x(s)) \mathbb{D}_{x} g(x(s))-\mathbb{D}_{x} f(x(s)) \mathbb{S}_{x} g(x(s))}{U_{2}(x(s))\left[\mathbb{D}_{x} g(x(s))\right]^{2}-\left[\mathbb{S}_{x} g(x(s))\right]^{2}} \\
\mathbb{S}_{x}\left(\frac{f(x(s))}{g(x(s))}\right) & =\frac{U_{2}(x(s)) \mathbb{D}_{x} f(x(s)) \mathbb{D}_{x} g(x(s))-\mathbb{S}_{x} f(x(s)) \mathbb{S}_{x} g(x(s))}{U_{2}(x(s))\left[\mathbb{D}_{x} g(x(s))\right]^{2}-\left[\mathbb{S}_{x} g(x(s))\right]^{2}},
\end{aligned}
$$

provided that $g(x(s)) \neq 0$.

3. More generally, relations (19)-(20) and (23)-(24) remain valid if we replace $x$ and $x_{1}$ by $x_{\mu}$ and $x_{\mu+1}$ respectively, $\mu \in \mathbb{C}$. In particular, the constant $\delta_{x}$ remains unchanged if we replace $x$ in (22) by $x_{k}, k \in \mathbb{Z}$, i.e.,

$$
\delta_{x_{k}}=\delta_{x}:=\delta, k \in \mathbb{Z}
$$

4. The operators $\mathbb{D}_{x}$ and $\mathbb{S}_{x}$ also satisfy the product rules II

$$
\begin{aligned}
\mathbb{D}_{x} \mathbb{S}_{x} & =\alpha \mathbb{S}_{x} \mathbb{D}_{x}+U_{1}(s) \mathbb{D}_{x}^{2} \\
\mathbb{S}_{x}^{2} & =U_{1}(s) \mathbb{S}_{x} \mathbb{D}_{x}+\alpha U_{2}(s) \mathbb{D}_{x}^{2}+\mathbb{I},
\end{aligned}
$$

where $\mathbb{I}$ is the identity operator $\mathbb{I} f(x)=f(x)$, and

$$
U_{1}(s):=U_{1}(x(s))=\left(\alpha^{2}-1\right) x(s)+\beta(\alpha+1), \quad U_{2}(s):=U_{2}(x(s)) .
$$




\subsection{Properties of the Basis $F_{n}$}

Looking for suitable bases for the companion operators, Foupouagnigni, Kenfack, Koepf, and Mboutngam [9] proved the following:

Theorem 2 [9]

The polynomial sequence

$$
F_{n}(x(s))=F_{n}\left(x(s), x\left(z_{x}\right)\right), \text { with } F_{n}(x(s), x(z))=\prod_{j=1}^{n}\left[x(s)-x_{j}(z)\right],
$$

where $z_{x}$ is the unique solution (provided that the lattice $x(s)$ is quadratic or q-quadratic: i.e. the constants $c_{j}$ in (10) satisfy $c_{1} c_{2} \neq 0$ or $c_{4} \neq 0$ ) in the variable $t$ of the equation

$$
x_{1}(t)=x(t)
$$

fulfills the following relations

$$
\begin{aligned}
\mathbb{D}_{x} F_{n}(x(s)) & =\gamma_{n} F_{n-1}(x(s)) \\
\mathbb{S}_{x} F_{n}(x(s)) & =\alpha_{n} F_{n}(x(s))+\frac{\gamma_{n}}{2} \nabla x_{n+1}\left(z_{x}\right) F_{n-1}(x(s)), \\
\mathbb{D}_{x} \frac{1}{F_{n}(x(s))} & =-\frac{\gamma_{n}}{F_{n+1}(x(s))} \\
\mathbb{S}_{x} \frac{1}{F_{n}(x(s))} & =\frac{\alpha_{n}}{F_{n}(x(s))}+\frac{\gamma_{n}}{2} \frac{\nabla x_{n+2}\left(z_{x}\right)}{F_{n+1}(x(s))}
\end{aligned}
$$

where $\alpha_{n}, \beta_{n}$ and $\gamma_{n}$ are defined in (8).

One straightforward corollary of the previous theorems is the following:

Corollary 1 The coefficients $\alpha_{n}$ and $\gamma_{n}$ fulfill the following relations

$$
\alpha_{n+1}=\alpha \alpha_{n}+\left(\alpha^{2}-1\right) \gamma_{n}, \gamma_{n+1}=\alpha_{n}+\alpha \gamma_{n},
$$

from which one deduces after some computations involving basic linear algebra that

$$
\alpha_{n}=1, \gamma_{n}=n, \text { for } \alpha=1,
$$

and

$$
\alpha_{n}=\frac{q^{\frac{n}{2}}+q^{-\frac{n}{2}}}{2}, \gamma_{n}=\frac{q^{\frac{n}{2}}-q^{-\frac{n}{2}}}{q^{\frac{1}{2}}-q^{-\frac{1}{2}}}, \text { for } \alpha=\frac{q^{\frac{1}{2}}+q^{-\frac{1}{2}}}{2} .
$$

Proof: This can easily be deduced by applying the operators $\mathbb{D}_{x}$ and $\mathbb{S}_{x}$ to both sides of the following relation deduced from (29)

$$
F_{n+1}(x(s))=\left(x(s)-x_{n+1}\left(z_{x}\right)\right) F_{n}(x(s)),
$$

and using the product rules (19) and (20).

Next, by considering, instead of the well-known Stieltjes function $S_{0}$ of the functional $\mathcal{L}$

$$
S_{0}[\mathcal{L}](z)=\sum_{n=0}^{\infty} \frac{\left\langle\mathcal{L}, x^{n}(s)\right\rangle}{x^{n+1}(z)}
$$

but rather its new representation [9] in terms of the appropriate basis $F_{n}$

$$
S[\mathcal{L}](z)=\sum_{n=0}^{\infty} \frac{\left\langle\mathcal{L}, F_{n}(x(s))\right\rangle}{F_{n+1}(x(z))}
$$

and using results of the previous theorems, the following result is proved in [9]. 
Theorem 3 [9]

$$
\begin{aligned}
S\left[\mathbb{D}_{x} \mathcal{L}\right](s) & =\mathbb{D}_{x}[S(\mathcal{L})](s), \\
S\left[\mathbb{S}_{x} \mathcal{L}\right](s) & =\alpha \mathbb{S}_{x} S[\mathcal{L}](s)+U_{1} \mathbb{D}_{x} S[\mathcal{L}](s) .
\end{aligned}
$$

Here, for a given $Q \in \mathbb{C}[x(s)], Q \mathcal{L}, \mathbb{D}_{x} \mathcal{L}$ and $\mathbb{S}_{x} \mathcal{L}$ are linear functionals defined as

$$
\langle Q \mathcal{L}, P\rangle=\langle\mathcal{L}, Q P\rangle,\left\langle\mathbb{D}_{x} \mathcal{L}, P\right\rangle=-\left\langle\mathcal{L}, \mathbb{D}_{x} P\right\rangle,\left\langle\mathbb{S}_{x} \mathcal{L}, P\right\rangle=\left\langle\mathcal{L}, \mathbb{S}_{x} P\right\rangle, \forall P \in \mathbb{C}[x(s)] .
$$

\section{Remark 1}

1. The functions $S_{0}[\mathcal{L}]$ and $S[\mathcal{L}]$ defined respectively by Equations (37) and (38) are equal. In fact, if the corresponding polynomial family is orthogonal with respect to a given positive measure $\mu(x)$, then the function $S_{0}$ reads

$$
S_{0}[\mathcal{L}](x(z))=\int_{\text {Supp. } \mu} \frac{d \mu(x(s))}{x(z)-x(s)} .
$$

The latter expression is equal to (38) by means of the relation (see [9], Corollary 7, page 6)

$$
\frac{1}{x(z)-x(s)}=\sum_{k=0}^{\infty} \frac{F_{k}(x(s))}{F_{k+1}(x(z))}, z \neq s .
$$

2. The expression $S(f \mathcal{L})$ can be evaluated using the well-known relation by Maroni [18]

$$
S[f \mathcal{L}](x)=f(x) S[\mathcal{L}](x)+\left(\mathcal{L} \theta_{0} f\right)(x), f \in \mathbb{C}[x],
$$

with

$$
\theta_{0} f(x)=\frac{f(x)-f(0)}{x},
$$

where the product of the functional $\mathcal{L}$ by a polynomial $g, \mathcal{L} g$, is defined as

$$
\mathcal{L} g(x(s))=\sum_{k=0}^{n} g_{k} \sum_{j=0}^{k}\left\langle\mathcal{L}, x^{j}(s)\right\rangle x^{k-j}(s), \quad \text { with } g(x(s))=\sum_{k=0}^{n} g_{k} x^{k}(s), n \geq 0 .
$$

\subsection{Pearson-type Equations}

Let $\left(P_{n}\right)_{n}$ be a family of COP on a non-uniform lattice. It is well-known that this polynomial sequence satisfies $[4,5,8]$ Equation (17). By assuming that $\mathcal{L}$ is the corresponding regular linear functional

$$
\left\langle\mathcal{L}, P_{n} P_{m}\right\rangle=k_{n} \delta_{n, m}, k_{n} \neq 0, \forall n, m \geq 0,
$$

we obtain using (17) and (41)

$$
\begin{aligned}
0 & =\langle\mathcal{L}, 0\rangle \\
& =\left\langle\mathcal{L}, \phi(x(s)) \mathbb{D}_{x}^{2} P_{n+1}(x(s))+\psi(x(s)) \mathbb{S}_{x} \mathbb{D}_{x} P_{n+1}(x(s))+\lambda_{n+1} P_{n+1}(x(s))\right\rangle, \forall n \geq 0 \\
& =\left\langle\mathbb{D}_{x}(\phi \mathcal{L})-\mathbb{S}_{x}(\psi \mathcal{L}), \mathbb{D}_{x} P_{n+1}(x(s))\right\rangle, \forall n \geq 0
\end{aligned}
$$

Since $\operatorname{deg}\left(\mathbb{D}_{x} P_{n+1}\right)=n, n \geq 0$, the sequence $\left(\mathbb{D}_{x} P_{n+1}(x(s))\right)_{n \geq 0}$ forms a basis of $\mathbb{C}[x(s)]$, therefore

$$
\mathbb{D}_{x}(\phi \mathcal{L})=\mathbb{S}_{x}(\psi \mathcal{L})
$$

\section{Definition 1}

We call (46) the Pearson equation for the linear functional $\mathcal{L}$ corresponding to the $C O P\left(P_{n}\right)_{n}$ satisfying (17). 


\section{Proposition 1}

Let $\left(P_{n}\right)_{n}$ be a polynomial family, orthogonal with respect to the weight function $\rho$ satisfying the Pearson equation (3) and the border conditions (5). Then, the linear functional $\mathcal{U}$ defined on $\mathbb{C}[x(s)]$ by

$$
\langle\mathcal{U}, P\rangle=\int_{C} \rho(s) P(x(s)) \nabla x_{1}(s) d s,
$$

for the orthogonality relation (1), where $C$ is an appropriate contour in the complex s-plane, or by

$$
\langle\mathcal{U}, P\rangle=\sum_{i=0}^{N} P\left(x\left(s_{i}\right)\right) \rho\left(s_{i}\right) \nabla x_{1}\left(s_{i}\right), N \in \mathbb{N} \cup\{+\infty\},
$$

for the orthogonality relation (2), satisfies the Pearson equation (46).

Proof: The proof uses the following relations obtained by direct computation taking into account the definitions of $\mathbb{D}_{x}$ and $\mathbb{S}_{x}$

$$
\begin{aligned}
\frac{\Delta}{\nabla x_{1}(s)}\left[f\left(s-\frac{1}{2}\right)\right] & =\mathbb{D}_{x} f(s) \\
\mathbb{D}_{x}(f(s) g(s)) & =f\left(s+\frac{1}{2}\right) \mathbb{D}_{x} g(s)+g\left(s-\frac{1}{2}\right) \mathbb{D}_{x} f(s) .
\end{aligned}
$$

In the first step, computations using (4), (41) and (47) for $P \in \mathbb{C}[x(s)]$ give

$$
\begin{aligned}
& \left\langle\mathbb{D}_{x}(\phi \mathcal{U})-\mathbb{S}_{x}(\psi \mathcal{U}), P\right\rangle=-\left\langle\mathcal{U}, \phi \mathbb{D}_{x} P+\psi \mathbb{S}_{x} P\right\rangle \\
= & -\int_{C} \rho(s)\left[\left(\sigma(s)+\frac{1}{2} \psi(x(s)) \nabla x_{1}(s)\right) \mathbb{D}_{x} P(x(s))+\psi(x(s)) \mathbb{S}_{x} P(x(s))\right] \nabla x_{1}(s) d s, \\
= & -\int_{C} \rho(s) \sigma(s) \mathbb{D}_{x} P(x(s)) \nabla x_{1}(s) d s \\
& -\int_{C} \psi(x(s)) \rho(s)\left[\frac{1}{2} \nabla x_{1}(s) \mathbb{D}_{x} P(x(s))+\mathbb{S}_{x} P(x(s))\right] \nabla x_{1}(s) d s .
\end{aligned}
$$

In the second step, we use (50) for $f(s)=P(x(s))$ and $g(s)=\sigma\left(s+\frac{1}{2}\right) \rho\left(s+\frac{1}{2}\right)$ and the relation

$$
\frac{1}{2} \nabla x_{1}(s) \mathbb{D}_{x} P(x(s))+\mathbb{S}_{x} P(x(s))=P\left(x\left(s+\frac{1}{2}\right)\right),
$$

which is easily deduced by direct computation, to transform (51) into

$$
\begin{aligned}
\left\langle\mathbb{D}_{x}(\phi \mathcal{U})-\mathbb{S}_{x}(\psi \mathcal{U}), P\right\rangle & =-\int_{C} \mathbb{D}_{x}\left[\sigma\left(s+\frac{1}{2}\right) \rho\left(s+\frac{1}{2}\right) P(x(s))\right] \nabla x_{1}(s) d s \\
& +\int_{C} P\left(x\left(s+\frac{1}{2}\right)\right) \mathbb{D}_{x}\left[\sigma\left(s+\frac{1}{2}\right) \rho\left(s+\frac{1}{2}\right)\right] \nabla x_{1}(s) d s \\
& -\int_{C} \psi(x(s)) \rho(s) P\left(x\left(s+\frac{1}{2}\right)\right) \nabla x_{1}(s) d s .
\end{aligned}
$$

In the third step we use the relation

$$
\mathbb{D}_{x}\left(\sigma\left(s+\frac{1}{2}\right) \rho\left(s+\frac{1}{2}\right)\right)=\psi(x(s)) \rho(s)
$$

which by means of (49) is equivalent to the Pearson equation (3), and the border conditions (5) to get

$$
\left\langle\mathbb{D}_{x}(\phi \mathcal{U})-\mathbb{S}_{x}(\psi \mathcal{U}), P\right\rangle=0 .
$$

The proof is similar if the linear functional $\mathcal{U}$ is represented by (48). 


\section{Characterization Theorem}

In this section, we first state and prove the following propositions, which are used to give the proof of the main results of this paper, stated in Theorem 4.

Proposition 2

The following relations hold for every linear functional $\mathcal{L}$ and for all polynomials $f$

and $g$.

$$
\begin{aligned}
\mathbb{D}_{x} \mathbb{S}_{x} f & =\frac{1}{\alpha} \mathbb{D}_{x}\left(U_{1}(s) \mathbb{D}_{x} f\right)+\frac{1}{\alpha} \mathbb{S}_{x} \mathbb{D}_{x} f \\
\mathbb{S}_{x}^{2} f & =\frac{1}{\alpha} \mathbb{S}_{x}\left(U_{1}(s) \mathbb{D}_{x} f\right)+\frac{1}{\alpha} U_{2}(s) \mathbb{D}_{x}^{2} f+f ; \\
f \mathbb{D}_{x} g & =\mathbb{D}_{x}\left[\left(\mathbb{S}_{x} f-\frac{U_{1}(x)}{\alpha} \mathbb{D}_{x} f\right) g\right]-\frac{1}{\alpha} \mathbb{S}_{x}\left(g \mathbb{D}_{x} f\right) \\
f \mathbb{S}_{x} g & =\mathbb{S}_{x}\left[\left(\mathbb{S}_{x} f-\frac{U_{1}(x)}{\alpha} \mathbb{D}_{x} f\right) g\right]-\frac{U_{2}(x)}{\alpha} \mathbb{D}_{x}\left(g \mathbb{D}_{x} f\right) ; \\
\left(\mathbb{D}_{x}^{2} f\right) g & =\mathbb{D}_{x}\left[\mathbb{D}_{x} f \mathbb{S}_{x} g-\mathbb{S}_{x} f \mathbb{D}_{x} g\right]+\left(\mathbb{D}_{x}^{2} g\right) f \\
\left(\mathbb{S}_{x} \mathbb{D}_{x} f\right) g & =\mathbb{S}_{x}\left[\mathbb{D}_{x} f \mathbb{S}_{x} g-\mathbb{S}_{x} f \mathbb{D}_{x} g\right]+\left(\mathbb{S}_{x} \mathbb{D}_{x} g\right) f .
\end{aligned}
$$

Proof: The proof of the first four relations is obtained by direct computation, starting from the right-hand side using relations (19), (20), (26), (27) and the following ones linking $U_{1}$ and $U_{2}$

$$
\mathbb{S}_{x}\left(U_{1}(x(s))=\alpha U_{1}\left(x(s), \mathbb{D}_{x}\left(U_{2}(x(s))=2 \alpha U_{1}\left(x(s), \mathbb{D}_{x} U_{1}(x(s))=\alpha^{2}-1 .\right.\right.\right.\right.
$$

Relations (56) and (57) are obtained by direct computation, starting from the right-hand side using relations (19), (20), (26), (27).

\section{Proposition 3}

The following relations hold for every linear functional $\mathcal{L}$ and for all polynomials $f, g, \phi$ and $\psi$.

$$
\begin{aligned}
\mathbb{D}_{x}(\phi \mathcal{L}) & =\mathbb{S}_{x}(\psi \mathcal{L}) \Longrightarrow\left\langle\mathcal{L},\left(\phi \mathbb{D}_{x}^{2} f+\psi \mathbb{S}_{x} \mathbb{D}_{x} f\right) g\right\rangle=\left\langle\mathcal{L},\left(\phi \mathbb{D}_{x}^{2} g+\psi \mathbb{S}_{x} \mathbb{D}_{x} g\right) f\right\rangle \\
\mathbb{D}_{x}(f \mathcal{L}) & =\left(\mathbb{S}_{x} f-\frac{U_{1}(s)}{\alpha} \mathbb{D}_{x} f\right) \mathbb{D}_{x} \mathcal{L}+\frac{1}{\alpha} \mathbb{D}_{x} f \mathbb{S}_{x} \mathcal{L} ; \\
\mathbb{S}_{x}(f \mathcal{L}) & =\left(\mathbb{S}_{x} f-\frac{U_{1}(s)}{\alpha} \mathbb{D}_{x} f\right) \mathbb{S}_{x} \mathcal{L}+\frac{1}{\alpha} \mathbb{D}_{x} f \mathbb{D}_{x}\left(U_{2} \mathcal{L}\right) ; \\
f \mathbb{D}_{x} \mathcal{L} & =\mathbb{D}_{x}\left(\mathbb{S}_{x} f \mathcal{L}\right)-\mathbb{S}_{x}\left(\mathbb{D}_{x} f \mathcal{L}\right) \\
f \mathbb{S}_{x} \mathcal{L} & =\mathbb{S}_{x}\left(\mathbb{S}_{x} f \mathcal{L}\right)-\mathbb{D}_{x}\left(U_{2} \mathbb{D}_{x} f \mathcal{L}\right) .
\end{aligned}
$$

Proof: Relation (59) is obtained by a straightforward application of (41), (56) and (57). Finally, Relations (60) and (61) are easily deduced from (54) and (55) respectively; while (62) and (63) are direct consequences of Relations (19) and (20).

Since the polynomial sequence $\left(Q_{n, m}\right)_{n \geq 0}$ fulfills $\operatorname{deg}\left(Q_{n, m}\right)=n, \forall n \in \mathbb{N}$, there exists [19] a sequence of linear functionals $\left(\hat{Q}_{n, m}\right)_{n \geq 0}$ called dual basis of $\left(Q_{n, m}\right)_{n \geq 0}$ satisfying

$$
\left\langle\hat{Q}_{n, m}, Q_{j, m}\right\rangle=\delta_{n, j}, n, j \geq 0 .
$$

Also, every linear functional $\mathcal{L}$ can be represented as [19]

$$
\mathcal{L}=\sum_{n=0}^{\infty}\left\langle\mathcal{L}, Q_{n, m}\right\rangle \hat{Q}_{n, m}
$$


In addition, if $\left(P_{n}\right)_{n}$ is a polynomial sequence orthogonal with respect to the linear functional $\mathcal{L}$, then its dual basis $\hat{P}_{n}$ is given by [12]

$$
\hat{P}_{n}=\frac{P_{n} \mathcal{L}}{\left\langle\mathcal{L}, P_{n} P_{n}\right\rangle}, n \geq 0 .
$$

The derivative of the dual basis $\hat{Q}_{n, m}$ fulfills

\section{Proposition 4}

$$
\mathbb{D}_{x} \widehat{Q}_{n, m}=-\gamma_{n+1} \widehat{Q}_{n+1, m-1} \quad \forall n \geq 0, \forall m \geq 1 .
$$

Proof: Using the following relation easily deduced from (71)-(72),

$$
Q_{n, m}=\frac{1}{\gamma_{n+1}} \mathbb{D}_{x} Q_{n+1, m-1}, \forall n \geq 0, m \geq 1,
$$

we obtain for fixed integers $n \geq 0$ and $m \geq 1$,

$$
\begin{aligned}
\left\langle\mathbb{D}_{x} \widehat{Q}_{n, m}, Q_{j+1, m-1}\right\rangle & =-\left\langle\widehat{Q}_{n, m}, \mathbb{D}_{x} Q_{j+1, m-1}\right\rangle, \forall j \geq 0 \\
& =-\left\langle\widehat{Q}_{n, m}, \gamma_{j+1} Q_{j, m}\right\rangle, \forall j \geq 0 \\
& =-\gamma_{n+1} \delta_{n, j}, \forall j \geq 0 \\
& =-\gamma_{n+1}\left\langle\widehat{Q}_{n+1, m-1}, Q_{j+1, m-1}\right\rangle, \forall j \geq 0 .
\end{aligned}
$$

In addition,

$$
\left\langle\mathbb{D}_{x} \widehat{Q}_{n, m}, Q_{0, m-1}\right\rangle=-\left\langle\widehat{Q}_{n, m}, \mathbb{D}_{x} Q_{0, m-1}\right\rangle=0=-\gamma_{n+1}\left\langle\widehat{Q}_{n+1, m-1}, Q_{0, m-1}\right\rangle .
$$

Therefore,

$$
\mathbb{D}_{x} \widehat{Q}_{n, m}=-\gamma_{n+1} \widehat{Q}_{n+1, m-1} .
$$

Proposition 5 Let $\mathcal{L}$ be a regular linear functional satisfying the Pearson equation

$$
\mathbb{D}_{x}(\phi \mathcal{L})=\mathbb{S}_{x}(\psi \mathcal{L}),
$$

where $\phi$ is a polynomial of degree at most 2 and $\psi$ a first degree polynomial. Then, we have

$$
\phi_{2} \gamma_{n}+\psi_{1} \alpha_{n} \neq 0, \forall n \geq 0,
$$

where $\phi_{2}$ and $\psi_{1}$ are the leading coefficients of the polynomials $\phi$ and $\psi$ with respect to the basis $\left(x^{n}(s)\right)_{n}$.

In addition, for any polynomial $P_{n}$ of degree $n$ in $x(s)$, we have

$$
\operatorname{deg}\left(\phi(x(s)) \mathbb{D}_{x}^{2} P_{n}(x(s))+\psi(x(s)) \mathbb{S}_{x} \mathbb{D}_{x} P_{n}(x(s))\right)=n, \forall n \geq 1 .
$$

Proof: Application of both sides of the Pearson equation to the polynomial $F_{n}$ yields the following difference equation for the moments $\hat{\mu}_{n}=\left\langle\mathcal{L}, F_{n}\right\rangle$

$$
\left(\phi_{2} \gamma_{n}+\psi_{1} \alpha_{n}\right) \hat{\mu}_{n+1}=u_{n} \hat{\mu}_{n}+v_{n} \hat{\mu}_{n-1},
$$

where $u_{n}$ and $v_{n}$ depend on $n$ and the coefficients of the polynomials $\phi$ and $\psi$. For all the moments to exist, property (69) is necessary. Relation (70) is easily deduced from (69) since if we write $P_{n}(x(s))=$ $a_{n} F_{n}(x(s))+\ldots, a_{n} \neq 0$, then we have

$$
\phi(x(s)) \mathbb{D}_{x}^{2} P_{n}(x(s))+\psi(x(s)) \mathbb{S}_{x} \mathbb{D}_{x} P_{n}(x(s))=a_{n} \gamma_{n}\left(\phi_{2} \gamma_{n-1}+\psi_{1} \alpha_{n-1}\right) F_{n}(x(s))+\ldots,
$$

with $a_{n} \gamma_{n}\left(\phi_{2} \gamma_{n-1}+\psi_{1} \alpha_{n-1}\right) \neq 0, \forall n \geq 1$. 


\section{Theorem 4}

Let $\mathcal{L}$ be a regular linear functional, $\left(P_{n}\right)_{n}$ its corresponding monic orthogonal polynomials and $Q_{n, m}$ the monic polynomial of degree $n$ defined by

$$
B_{n, m} Q_{n, m}=\mathbb{D}_{x}^{m} P_{n+m}, m, n \geq 0,
$$

with

$$
B_{n, m}=\prod_{j=0}^{m-1} \gamma_{n+m-j}=\frac{\gamma_{n+m} !}{\gamma_{n} !}, \quad Q_{n, 0} \equiv P_{n} .
$$

The following properties are equivalent:

(a) There exist two polynomials, $\phi$ of degree at most two and $\psi$ of degree one, such that

$$
\mathbb{D}_{x}(\phi \mathcal{L})=\mathbb{S}_{x}(\psi \mathcal{L})
$$

(b) There exist two polynomials, $\phi$ of degree at most two and $\psi$ of degree one, such that for any integer $m \geq 0$

$$
\begin{aligned}
& \mathbb{D}_{x}\left(\phi^{(m)} \mathcal{L}_{m}\right)=\mathbb{S}_{x}\left(\psi^{(m)} \mathcal{L}_{m}\right), \\
& \left\langle\mathcal{L}_{m}, Q_{n, m} Q_{j, m}\right\rangle=k_{n} \delta_{j, n}, k_{n} \neq 0, \forall n, j \in \mathbb{N},
\end{aligned}
$$

where the linear functional $\mathcal{L}_{m}$ and the polynomials $\phi^{(m)}$ and $\psi^{(m)}$ are defined respectively by

$$
\begin{aligned}
& \phi^{(m+1)}=\mathbb{S}_{x} \phi^{(m)}+U_{1} \mathbb{S}_{x} \psi^{(m)}+\alpha U_{2} \mathbb{D}_{x} \psi^{(m)}, \quad \phi^{(0)} \equiv \phi \\
& \psi^{(m+1)}=\mathbb{D}_{x} \phi^{(m)}+\alpha \mathbb{S}_{x} \psi^{(m)}+U_{1} \mathbb{D}_{x} \psi^{(m)}, \quad \psi^{(0)} \equiv \psi, \\
& \mathcal{L}_{m+1}=\mathbb{D}_{x}\left[U_{2} \psi^{(m)} \mathcal{L}_{m}\right]-\mathbb{S}_{x}\left[\phi^{(m)} \mathcal{L}_{m}\right], \quad \mathcal{L}_{0} \equiv \mathcal{L},
\end{aligned}
$$

with the polynomials $U_{2}$ and $U_{1}$ given respectively by (21) and (28).

(c) There exist two polynomials, $\phi$ of degree at most two and $\psi$ of degree one, such that for any integer $m \geq 0$ the following second-order difference equation holds:

$$
\phi^{(m)}(x(s)) \mathbb{D}_{x}^{2} Q_{n, m}(x(s))+\psi^{(m)}(x(s)) \mathbb{S}_{x} \mathbb{D}_{x} Q_{n, m}(x(s))+\lambda_{n, m} Q_{n, m}(x(s))=0, \forall n \geq 0,
$$

where the polynomials $\phi^{(m)}$ and $\psi^{(m)}$ are given by (76), (77) and the constant

$$
\lambda_{n, m}=-\gamma_{n}\left\{\phi_{2}^{(m)} \gamma_{n-1}+\psi_{1}^{(m)} \alpha_{n-1}\right\}
$$

with

$$
\phi^{(m)}(x(s))=\phi_{2}^{(m)} x^{2}(s)+\phi_{1}^{(m)} x(s)+\phi_{0}^{(m)}, \psi^{(m)}(x(s))=\psi_{1}^{(m)} x(s)+\psi_{0}^{(m)},
$$

where the polynomials $\phi^{(m)}$ and $\psi^{(m)}$ are defined in (76)-(77).

(d) There exist two polynomials, $\phi$ of degree at most two and $\psi$ of degree one, such that for any integer $m \geq 0$ the following Rodrigues relation holds:

$$
\gamma_{n} \mathbb{D}_{x}\left(Q_{n-1, m+1} \mathcal{L}_{m+1}\right)=\alpha \lambda_{n, m} Q_{n, m} \mathcal{L}_{m}, \forall n \geq 1,
$$

where $\mathcal{L}_{m}$ is defined by Equations (76)-(78), and $\lambda_{n, m}$ defined by (80), with the initial condition

$$
\langle\mathcal{L}, \psi\rangle=0 .
$$


(e) There exist two polynomials, $\phi$ of degree at most two and $\psi$ of degree one, such that for any integer $m \geq 0$, there exist three sequences $\left(a_{n, n+1}^{m}\right)_{n},\left(a_{n, n}^{m}\right)_{n}$ and $\left(a_{n, n-1}^{m}\right)_{n}$, such that the so-called first structure relation is satisfied:

$$
\psi^{(m)} \mathbb{S}_{x}^{2} Q_{n, m}+\phi^{(m)} \mathbb{D}_{x} \mathbb{S}_{x} Q_{n, m}=a_{n, n+1}^{m} Q_{n+1, m}+a_{n, n}^{m} Q_{n, m}+a_{n, n-1}^{m} Q_{n-1, m}, \forall n \geq 1,
$$

with $a_{n, n-1}^{m} \neq 0$ for $n>2$, where the polynomials $\phi^{(m)}$ and $\psi^{(m)}$ are defined in (76)-(77).

(f) For any integer $m \geq 0$, there exist three sequences $\left(b_{n, n+1}^{m}\right)_{n},\left(b_{n, n}^{m}\right)_{n}$ and $\left(b_{n, n-1}^{m}\right)_{n}$, such that the following relation, called second structure relation, is satisfied:

$$
\mathbb{S}_{x} Q_{n, m}=b_{n, n+1}^{m} \mathbb{D}_{x} Q_{n+1, m}+b_{n, n}^{m} \mathbb{D}_{x} Q_{n, m}+b_{n, n-1}^{m} \mathbb{D}_{x} Q_{n-1, m}, \forall n \geq 1,
$$

with $b_{n, n-1}^{m} \neq 0$ for $n>2$.

(g) There exist three polynomials, $A, B$ and $C$ of degree at most two, one and zero respectively such that the following Riccati equation for the formal Stieltjes function $S(\mathcal{L}):=S$ of the linear functional $\mathcal{L}$ is satisfied

$$
A(x(s)) \mathbb{D}_{x}(S(\mathcal{L}))=B(x(s)) \mathbb{S}_{x}(S(\mathcal{L}))+C .
$$

\section{Proof: Proof of Theorem 1}

We organize the proof in the following scheme:

Step 1: $(a) \Rightarrow(b) \Rightarrow(c) \Rightarrow(a)$ which is equivalent to $(a) \Leftrightarrow(b) \Leftrightarrow(c)$.

Step 2: $(b)+(c) \Rightarrow(d) \Rightarrow(a)$ which taking into account Step 1, is equivalent to $(c) \Leftrightarrow(d)$.

Step 3: $(a)+(b)+(c) \Rightarrow(f) \Rightarrow(a)$ which using Step 1, is equivalent to $(a) \Leftrightarrow(f)$.

Step 4: $(c)+(f) \Rightarrow(e) \Rightarrow(a)$ which thanks to Step 3 is equivalent to $(e) \Leftrightarrow(f)$.

Step 5: $(a) \Leftrightarrow(g)$.

Step 1: $(a) \Rightarrow(b) \Rightarrow(c) \Rightarrow(a)$

Step 1.1 $(a) \Rightarrow(b)$.

We assume that the property $(a)$ is satisfied and we show by induction on $m$ that (74) and (75) are satisfied for $m \geq 0$. ¿From the Pearson equation (73) and the orthogonality relation (44) for the family $\left(P_{n}\right)$, it is obvious that the relations (74) and (75) are satisfied for $m=0$. Assume that they are satisfied up to a fixed integer $m>0$. Firstly, we use Relation (68) and the definition of $\mathcal{L}_{m+1}$ given by (78) to get for $0 \leq j \leq n$,

$$
\begin{aligned}
& \left\langle\mathcal{L}_{m+1}, Q_{n, m+1} Q_{j, m+1}\right\rangle=-\left\langle\mathcal{L}_{m}, \psi^{(m)} U_{2} \mathbb{D}_{x}\left(Q_{n, m+1} Q_{j, m+1}\right)+\phi^{(m)} \mathbb{S}_{x}\left(Q_{n, m+1} Q_{j, m+1}\right)\right\rangle \\
= & -\frac{1}{\gamma_{n+1} \gamma_{j+1}}\left\langle\psi^{(m)} \mathcal{L}_{m}, U_{2} \mathbb{D}_{x}\left(\mathbb{D}_{x} Q_{n+1, m} \mathbb{D}_{x} Q_{j+1, m}\right)\right\rangle+\left\langle\phi^{(m)} \mathcal{L}_{m}, \mathbb{S}_{x}\left(\mathbb{D}_{x} Q_{n+1, m} \mathbb{D}_{x} Q_{j+1, m}\right)\right\rangle .
\end{aligned}
$$

Secondly, we use (54) and (55) for $f=Q_{n+1, m}$ and $g=\mathbb{D}_{x} Q_{j+1, m}$

$$
\begin{aligned}
\mathbb{S}_{x}\left(\mathbb{D}_{x} Q_{j+1, m} \mathbb{D}_{x} Q_{n+1, m}\right)= & -\alpha Q_{n+1, m} \mathbb{D}_{x}^{2} Q_{j+1, m} \\
& +\alpha \mathbb{D}_{x}\left[\left(\mathbb{S}_{x} Q_{n+1, m}-\frac{U_{1}(x)}{\alpha} \mathbb{D}_{x} Q_{n+1, m}\right) \mathbb{D}_{x} Q_{j+1, m}\right], \\
U_{2}(x) \mathbb{D}_{x}\left(\mathbb{D}_{x} Q_{j+1, m} \mathbb{D}_{x} Q_{n+1, m}\right)= & -\alpha Q_{n+1, m} \mathbb{S}_{x} \mathbb{D}_{x} Q_{j+1, m} \\
& +\alpha \mathbb{S}_{x}\left[\left(\mathbb{S}_{x} Q_{n+1, m}-\frac{U_{1}(x)}{\alpha} \mathbb{D}_{x} Q_{n+1, m}\right) \mathbb{D}_{x} Q_{j+1, m}\right],
\end{aligned}
$$

to obtain after making use of the Pearson equation (74)

$$
\left\langle\mathcal{L}_{m+1}, Q_{n, m+1} Q_{j, m+1}\right\rangle=\frac{\alpha}{\gamma_{n+1} \gamma_{j+1}}\left\langle\mathcal{L}_{m}, Q_{n+1, m}\left(\phi^{(m)} \mathbb{D}_{x}^{2} Q_{j+1, m}+\psi^{(m)} \mathbb{S}_{x} \mathbb{D}_{x} Q_{j+1, m}\right)\right\rangle .
$$


Combination of (74), (75) and Proposition 5 lead to

$$
\begin{aligned}
\left\langle\mathcal{L}_{m+1}, Q_{n, m+1} Q_{j, m+1}\right\rangle & =\frac{\alpha\left(\gamma_{j} \phi_{2}^{(m)}+\alpha_{j} \psi_{1}^{(m)}\right)}{\gamma_{n+1}}\left\langle\mathcal{L}_{m}, Q_{n+1, m}^{2}\right\rangle \delta_{j, n}, 0 \leq j \leq n \\
& \neq 0, \text { for } j=n,
\end{aligned}
$$

thanks to Proposition 5 with $\mathcal{L}$ replaced by $\mathcal{L}_{m}$.

Next, we show that (74) is satisfied at order $m+1$, using mainly the fact it is satisfied at order $m$. Let $P$ be a polynomial of degree at least 1 . We have

$$
\begin{aligned}
I & =\left\langle-\mathbb{D}_{x}\left(\phi^{(m+1)} \mathcal{L}_{m+1}\right)+\mathbb{S}_{x}\left(\psi^{(m+1)} \mathcal{L}_{m+1}\right), \mathbb{D}_{x} P\right\rangle \\
& \left.=\left\langle\mathcal{L}_{m+1},\left(\phi^{(m+1)} \mathbb{D}_{x}^{2} P+\psi^{(m+1)} \mathbb{S}_{x} \mathbb{D}_{x} P\right)\right)\right\rangle \\
& \left.=\left\langle\mathbb{D}_{x}\left(U_{2} \psi^{(m)} \mathcal{L}_{m}\right)-\mathbb{S}_{x}\left(\phi^{(m)} \mathcal{L}_{m}\right),\left(\phi^{(m+1)} \mathbb{D}_{x}^{2} P+\psi^{(m+1)} \mathbb{S}_{x} \mathbb{D}_{x} P\right)\right)\right\rangle .
\end{aligned}
$$

Use of relations (52) and (53) for $f=P$, the previous relation becomes:

$$
\begin{aligned}
I= & \left\langle\psi^{(m)} \mathcal{L}_{m},-U_{2} \mathbb{D}_{x}\left[\phi^{(m+1)} \mathbb{D}_{x}^{2} P+\psi^{(m+1)}\left[\alpha \mathbb{D}_{x} \mathbb{S}_{x} P-\mathbb{D}_{x}\left(U_{1} \mathbb{D}_{x} P\right)\right]\right]\right. \\
& +\left\langle\phi^{(m)} \mathcal{L}_{m},-\mathbb{S}_{x}\left[\phi^{(m+1)} \mathbb{D}_{x}^{2} P+\psi^{(m+1)}\left[\alpha \mathbb{D}_{x} \mathbb{S}_{x} P-\mathbb{D}_{x}\left(U_{1} \mathbb{D}_{x} P\right)\right]\right]\right\rangle \\
= & \left\langle\psi^{(m)} \mathcal{L}_{m},-U_{2} \mathbb{D}_{x}\left[\phi^{(m+1)} \mathbb{D}_{x}^{2} P\right]\right\rangle+\left\langle\psi^{(m)} \mathcal{L}_{m},-U_{2} \mathbb{D}_{x}\left[\psi^{(m+1)} \mathbb{D}_{x}\left[\alpha \mathbb{S}_{x} P-U_{1} \mathbb{D}_{x} P\right]\right]\right. \\
& +\left\langle\phi^{(m)} \mathcal{L}_{m},-\mathbb{S}_{x}\left[\phi^{(m+1)} \mathbb{D}_{x}^{2} P\right]\right\rangle+\left\langle\phi^{(m)} \mathcal{L}_{m},-\mathbb{S}_{x}\left[\psi^{(m+1)} \mathbb{D}_{x}\left[\alpha \mathbb{S}_{x} P-U_{1} \mathbb{D}_{x} P\right]\right]\right\rangle
\end{aligned}
$$

Using (54) and (55), first for $f=\mathbb{D}_{x} P, g=\phi^{(m+1)}$, then again for $f=\alpha \mathbb{S}_{x} P-U_{1} \mathbb{D}_{x} P, g=\psi^{(m+1)}$, we obtain after making use of the Pearson equation (74):

$$
\begin{aligned}
I= & \left\langle\psi^{(m)} \mathcal{L}_{m}, \mathbb{D}_{x} P \mathbb{S}_{x} \phi^{(m+1)}+\left(\alpha \mathbb{S}_{x} P-U_{1} \mathbb{D}_{x} P\right) \mathbb{S}_{x} \psi^{(m+1)}\right\rangle \\
& +\left\langle\phi^{(m)} \mathcal{L}_{m}, \mathbb{D}_{x} P \mathbb{D}_{x} \phi^{(m+1)}+\left(\alpha \mathbb{S}_{x} P-U_{1} \mathbb{D}_{x} P\right) \mathbb{D}_{x} \psi^{(m+1)}\right\rangle .
\end{aligned}
$$

By remarking that

$$
\mathbb{S}_{x} U_{1}=\alpha U_{1}, \mathbb{D}_{x} U_{2}=2 \alpha U_{1}, \mathbb{D}_{x} U_{1}=\alpha^{2}-1, \mathbb{S}_{x} U_{2}=\alpha^{2} U_{2}+U_{1}^{2}, \mathbb{D}_{x}^{2} \psi^{(m)}=0,
$$

we get after some computation using (19), (20), (26), (27), (76) and (77)

$$
\begin{aligned}
\mathbb{S}_{x} \phi^{(m+1)}= & U_{1} \mathbb{S}_{x} \mathbb{D}_{x} \phi^{(m)}+\alpha U_{2} \mathbb{D}_{x}^{2} \phi^{(m)}+\phi^{(m)}+\alpha U_{1} \psi^{(m)} \\
& +\left[2 \alpha U_{1}^{2}+\alpha\left(2 \alpha^{2}-1\right) U_{2}\right] \mathbb{S}_{x} \mathbb{D}_{x} \psi^{(m)} \\
\mathbb{S}_{x} \psi^{(m+1)}= & \mathbb{S}_{x} \mathbb{D}_{x} \phi^{(m)}+2 \alpha U_{1} \mathbb{S}_{x} \mathbb{D}_{x} \psi^{(m)}+\alpha \psi^{(m)} \\
\mathbb{D}_{x} \phi^{(m+1)}= & \alpha \mathbb{S}_{x} \mathbb{D}_{x} \phi^{(m)}+U_{1} \mathbb{D}_{x}^{2} \phi^{(m)}+\left(4 \alpha^{2}-1\right) U_{1} \mathbb{S}_{x} \mathbb{D}_{x} \psi^{(m)}+\left(\alpha^{2}-1\right) \psi^{(m)}, \\
\mathbb{D}_{x} \psi^{(m+1)}= & \mathbb{D}_{x}^{2} \phi^{(m)}+\left(2 \alpha^{2}-1\right) \mathbb{S}_{x} \mathbb{D}_{x} \psi^{(m)} .
\end{aligned}
$$

Subtituting (88) into (87) we get after using the product rules (19) and (20)

$$
\begin{aligned}
I= & \left\langle\psi^{(m)} \mathcal{L}_{m}, \mathbb{D}_{x} P\left(\alpha U_{2} \mathbb{D}_{x}^{2} \phi^{(m)}+\alpha\left(2 \alpha^{2}-1\right) U_{2} \mathbb{S}_{x} \mathbb{D}_{x} \psi^{(m)}+\phi^{(m)}\right)\right\rangle \\
& +\left\langle\psi^{(m)} \mathcal{L}_{m}, \alpha \mathbb{S}_{x} P\left(\mathbb{S}_{x} \mathbb{D}_{x} \phi^{(m)}+2 \alpha U_{1} \mathbb{S}_{x} \mathbb{D}_{x} \psi^{(m)}+\alpha \psi^{(m)}\right)\right\rangle \\
& +\left\langle\phi^{(m)} \mathcal{L}_{m}, \mathbb{D}_{x} P\left(\alpha \mathbb{S}_{x} \mathbb{D}_{x} \phi^{(m)}+2 \alpha^{2} U_{1} \mathbb{S}_{x} \mathbb{D}_{x} \psi^{(m)}+\alpha^{2} \psi^{(m)}-\psi^{(m)}\right)\right\rangle \\
& +\left\langle\phi^{(m)} \mathcal{L}_{m}, \alpha \mathbb{S}_{x} P\left(\mathbb{D}_{x}^{2} \phi^{(m)}+\left(2 \alpha^{2}-1\right) \mathbb{S}_{x} \mathbb{D}_{x} \psi^{(m)}\right)\right\rangle \\
= & \left\langle\psi^{(m)} \mathcal{L}_{m}, \alpha\left(\mathbb{S}_{x} P \mathbb{S}_{x} \mathbb{D}_{x} \phi^{(m)}+U_{2} \mathbb{D}_{x} P \mathbb{D}_{x}^{2} \phi^{(m)}\right)+\alpha\left(2 \alpha^{2}-1\right) U_{2} \mathbb{D}_{x} P \mathbb{S}_{x} \mathbb{D}_{x} \psi^{(m)}\right\rangle
\end{aligned}
$$




$$
\begin{aligned}
+ & \left\langle\psi^{(m)} \mathcal{L}_{m}, \alpha \mathbb{S}_{x} P\left(2 \alpha U_{1} \mathbb{S}_{x} \mathbb{D}_{x} \psi^{(m)}+\alpha \psi^{(m)}\right)\right\rangle \\
+ & \left\langle\phi^{(m)} \mathcal{L}_{m}, \alpha\left(\mathbb{S}_{x} P \mathbb{D}_{x}^{2} \phi^{(m)}+\mathbb{D}_{x} P \mathbb{S}_{x} \mathbb{D}_{x} \phi^{(m)}\right)+\alpha \mathbb{D}_{x} P\left(2 \alpha U_{1} \mathbb{S}_{x} \mathbb{D}_{x} \psi^{(m)}+\alpha \psi^{(m)}\right)\right\rangle \\
& +\left\langle\phi^{(m)} \mathcal{L}_{m}, \alpha\left(2 \alpha^{2}-1\right) \mathbb{S}_{x} P \mathbb{S}_{x} \mathbb{D}_{x} \psi^{(m)}\right\rangle \\
= & \left\langle\psi^{(m)} \mathcal{L}_{m}, \alpha \mathbb{S}_{x}\left(P \mathbb{D}_{x} \phi^{(m)}\right)+\alpha\left(2 \alpha^{2}-1\right) U_{2} \mathbb{D}_{x} P \mathbb{S}_{x} \mathbb{D}_{x} \psi^{(m)}+\alpha \mathbb{S}_{x} P\left(2 \alpha U_{1} \mathbb{S}_{x} \mathbb{D}_{x} \psi^{(m)}+\alpha \psi^{(m)}\right)\right\rangle \\
& +\left\langle\phi^{(m)} \mathcal{L}_{m}, \alpha \mathbb{D}_{x}\left(P \mathbb{D}_{x} \phi^{(m)}\right)+\alpha \mathbb{D}_{x} P\left(2 \alpha U_{1} \mathbb{S}_{x} \mathbb{D}_{x} \psi^{(m)}+\alpha \psi^{(m)}\right)+\alpha\left(2 \alpha^{2}-1\right) \mathbb{S}_{x} P \mathbb{S}_{x} \mathbb{D}_{x} \psi^{(m)}\right\rangle .
\end{aligned}
$$

Use of the Pearson equation (74) allows to get from the previous equation:

$$
\begin{aligned}
I= & \left\langle\psi^{(m)} \mathcal{L}_{m}, \alpha\left(2 \alpha^{2}-1\right) U_{2} \mathbb{D}_{x} P \mathbb{S}_{x} \mathbb{D}_{x} \psi^{(m)}+\alpha \mathbb{S}_{x} P\left(2 \alpha U_{1} \mathbb{S}_{x} \mathbb{D}_{x} \psi^{(m)}+\alpha \psi^{(m)}\right)\right\rangle \\
& +\left\langle\phi^{(m)} \mathcal{L}_{m}, \alpha \mathbb{D}_{x} P\left(2 \alpha U_{1} \mathbb{S}_{x} \mathbb{D}_{x} \psi^{(m)}+\alpha \psi^{(m)}\right)+\alpha\left(2 \alpha^{2}-1\right) \mathbb{S}_{x} P \mathbb{S}_{x} \mathbb{D}_{x} \psi^{(m)}\right\rangle
\end{aligned}
$$

Taking into account the following relations which can easily be deduced from (19), (20), (26) and (27),

$$
\begin{aligned}
\mathbb{S}_{x}\left(U_{1} \mathbb{D}_{x} \psi^{(m)}+\alpha \mathbb{S}_{x} \psi^{(m)}\right) & =2 \alpha U_{1} \mathbb{S}_{x} \mathbb{D}_{x} \psi^{(m)}+\alpha \psi^{(m)}, \\
\mathbb{D}_{x}\left(U_{1} \mathbb{D}_{x} \psi^{(m)}+\alpha \mathbb{S}_{x} \psi^{(m)}\right) & =\left(2 \alpha^{2}-1\right) \mathbb{S}_{x} \mathbb{D}_{x} \psi^{(m)}
\end{aligned}
$$

we get from the Equation (89, after using again the Pearson equation at the order $m$ :

$$
\begin{aligned}
I= & \alpha\left\langle\psi^{(m)} \mathcal{L}_{m}, U_{2} \mathbb{D}_{x} P \mathbb{D}_{x}\left(\alpha \mathbb{S}_{x} \psi^{(m)}+U_{1} \mathbb{D}_{x} \psi^{(m)}\right)+\mathbb{S}_{x} P \mathbb{S}_{x}\left(\alpha \mathbb{S}_{x} \psi^{(m)}+U_{1} \mathbb{D}_{x} \psi^{(m)}\right)\right\rangle \\
& \alpha\left\langle\phi^{(m)} \mathcal{L}_{m}, \mathbb{D}_{x} P \mathbb{S}_{x}\left(\alpha \mathbb{S}_{x} \psi^{(m)}+U_{1} \mathbb{D}_{x} \psi^{(m)}\right)+\mathbb{S}_{x} P \mathbb{D}_{x}\left(\alpha \mathbb{S}_{x} \psi^{(m)}+U_{1} \mathbb{D}_{x} \psi^{(m)}\right)\right\rangle . \\
= & \alpha\left\langle\psi^{(m)} \mathcal{L}_{m}, \mathbb{S}_{x}\left(P\left(U_{1} \mathbb{D}_{x} \psi^{(m)}+\alpha \mathbb{S}_{x} \psi^{(m)}\right)\right)\right\rangle+\alpha\left\langle\phi^{(m)} \mathcal{L}_{m}, \mathbb{D}_{x}\left(P\left(U_{1} \mathbb{D}_{x} \psi^{(m)}+\alpha \mathbb{S}_{x} \psi^{(m)}\right)\right)\right\rangle \\
= & 0 .
\end{aligned}
$$

Therefore,

$$
\mathbb{D}_{x}\left(\phi^{(m+1)} \mathcal{L}_{m+1}\right)=\mathbb{S}_{x}\left(\psi^{(m+1)} \mathcal{L}_{m+1}\right)
$$

Step 1.2 $(b) \Rightarrow(c)$.

We assume $(b)$ and fix two nonnegative integers $n$ and $m$. Then from the following expansion

$$
\phi^{(m)} \mathbb{D}_{x}^{2} Q_{n, m}+\psi^{(m)} \mathbb{S}_{x} \mathbb{D}_{x} Q_{n, m}=\sum_{j=0}^{n} a_{n, j} Q_{j, m}
$$

we deduce for $0 \leq k \leq n$

$$
a_{n, k}\left\langle\mathcal{L}_{m}, Q_{k, m}^{2}\right\rangle=\left\langle\mathcal{L}_{m},\left(\phi^{(m)} \mathbb{D}_{x}^{2} Q_{n, m}+\psi^{(m)} \mathbb{S}_{x} \mathbb{D}_{x} Q_{n, m}\right) Q_{k, m}\right\rangle
$$

Next, taking into account (74), we use the property (59) for $\phi=\phi^{(m)}, \psi=\psi^{(m)}, f=Q_{n, m}$ and $g=Q_{k, m}$ to obtain

$$
a_{n, k}\left\langle\mathcal{L}_{m}, Q_{k, m}^{2}\right\rangle=\left\langle\mathcal{L}_{m},\left(\phi^{(m)} \mathbb{D}_{x}^{2} Q_{k, m}+\psi^{(m)} \mathbb{S}_{x} \mathbb{D}_{x} Q_{k, m}\right) Q_{n, m}\right\rangle
$$

Therefore, since $\phi^{(m)} \mathbb{D}_{x}^{2} Q_{k, m}+\psi^{(m)} \mathbb{S}_{x} \mathbb{D}_{x} Q_{k, m}$ is a polynomial of degree at most $k$, we get

$$
a_{n, k}=0, \text { for } k<n .
$$

Finally, we write in (90) $Q_{n, m}=F_{n}(x(s))+$ lower terms and identify the coefficient of $F_{n}$ on both sides of (90) using relations (30) and (31) to get $a_{n, m}=-\lambda_{n, m}$ given by (80). 
Step $1.3(c) \Rightarrow(a)$.

We assume Property $(c)$ and obtain for fixed $n \geq 0$ and $m=1$ after taking into account (79)

$$
\begin{aligned}
\left\langle\mathbb{D}_{x}(\phi \mathcal{L})-\mathbb{S}_{x}(\phi \mathcal{L}), Q_{n, 1}\right\rangle & =\frac{1}{\gamma_{n+1}}\left\langle\mathbb{D}_{x}(\phi \mathcal{L})-\mathbb{S}_{x}(\phi \mathcal{L}), \mathbb{D}_{x} P_{n+1}\right\rangle \\
& =\frac{-1}{\gamma_{n+1}}\left\langle\mathcal{L}, \phi \mathbb{D}_{x}^{2} P_{n+1}+\psi \mathbb{S}_{x} \mathbb{D}_{x} P_{n+1}\right\rangle \\
& =\frac{\lambda_{n+1,0}}{\gamma_{n+1}}\left\langle\mathcal{L}, P_{n+1}\right\rangle=0 .
\end{aligned}
$$

Since $\left(Q_{n, 1}\right)_{n}$ forms a basis of $\mathbb{C}[x]$, we deduce that $\mathbb{D}_{x}(\phi \mathcal{L})=\mathbb{S}_{x}(\psi \mathcal{L})$.

Step 2: $(c) \Leftrightarrow(d)$.

Step 2.1 $(b)+(c) \Rightarrow(d)$.

We assume property $(c)$. Since we have established the above equivalence between properties $(a),(b)$ and $(c)$, we can then in addition make use of property $(b)$. Let $P \in \mathbb{C}[x]$. Using Relations (78) and (68), we obtain for fixed integers $n \geq 1$ and $m \geq 0$

$$
\begin{aligned}
\left\langle\mathbb{D}_{x}\left(Q_{n-1, m+1} \mathcal{L}_{m+1}\right), P\right\rangle & =-\left\langle Q_{n-1, m+1} \mathcal{L}_{m+1}, \mathbb{D}_{x} P\right\rangle \\
& =-\left\langle\mathcal{L}_{m+1}, Q_{n-1, m+1} \mathbb{D}_{x} P\right\rangle \\
& =-\left\langle\mathbb{D}_{x}\left(U_{2} \psi^{(m)} \mathcal{L}_{m}\right)-\mathbb{S}_{x}\left(\phi^{(m)} \mathcal{L}_{m}\right), \frac{\mathbb{D}_{x} Q_{n, m}}{\gamma_{n}} \mathbb{D}_{x} P\right\rangle \\
& =\frac{1}{\gamma_{n}}\left\langle\mathcal{L}_{m}, \psi^{(m)} U_{2} \mathbb{D}_{x}\left(\mathbb{D}_{x} Q_{n, m} \mathbb{D}_{x} P\right)+\phi^{(m)} \mathbb{S}_{x}\left(\mathbb{D}_{x} Q_{n, m} \mathbb{D}_{x} P\right)\right\rangle
\end{aligned}
$$

Use of the relations (54) and (55) for $f=P$ and $g=\mathbb{D}_{x} Q_{n, m}$

$$
\begin{aligned}
U_{2} \mathbb{D}_{x}\left(\mathbb{D}_{x} Q_{n, m} \mathbb{D}_{x} P\right) & =-\alpha P \mathbb{S}_{x} \mathbb{D}_{x} Q_{n, m}+\alpha \mathbb{S}_{x}\left[\left(\mathbb{S}_{x} P-\frac{U_{1}}{\alpha} \mathbb{D}_{x} P\right) \mathbb{D}_{x} Q_{n, m}\right], \\
\mathbb{S}_{x}\left(\mathbb{D}_{x} Q_{n, m} \mathbb{D}_{x} P\right) & =-\alpha P \mathbb{D}_{x}^{2} Q_{n, m}+\alpha \mathbb{D}_{x}\left[\left(\mathbb{S}_{x} P-\frac{U_{1}}{\alpha} \mathbb{D}_{x} P\right) \mathbb{D}_{x} Q_{n, m}\right]
\end{aligned}
$$

together with the Pearson equation for $\mathcal{L}_{m}$, namely (74), transform the previous equation into

$$
\left\langle\gamma_{n} \mathbb{D}_{x}\left(Q_{n-1, m+1} \mathcal{L}_{m+1}\right), P\right\rangle=-\alpha\left\langle\mathcal{L}_{m},\left(\phi^{(m)} \mathbb{D}_{x}^{2} Q_{n, m}+\psi^{(m)} \mathbb{S}_{x} \mathbb{D}_{x} Q_{n, m}\right) P\right\rangle .
$$

By means of (79), the latter equation reads

$$
\left\langle\gamma_{n} \mathbb{D}_{x}\left(Q_{n-1, m+1} \mathcal{L}_{m+1}\right), P\right\rangle=\alpha \lambda_{n, m}\left\langle Q_{n, m} \mathcal{L}_{m}, P\right\rangle
$$

Thus, we have

$$
\gamma_{n} \mathbb{D}_{x}\left(Q_{n-1, m+1} \mathcal{L}_{m+1}\right)=\alpha \lambda_{n, m} Q_{n, m} \mathcal{L}_{m}
$$

Equation (79) for $n=1$ and $m=0$ gives $\psi+\lambda_{1} P_{1}=0$. Therefore, $\langle\mathcal{L}, \psi\rangle=-\lambda_{1}\left\langle\mathcal{L}, P_{1}\right\rangle=0$.

Step 2.2 $(d) \Rightarrow(c)$.

We assume that the property $(d)$ is satisfied. Since $(a) \Longleftrightarrow(b) \Longleftrightarrow(c)$, We will show that $(a)$ is satisfied.

First, we use Equation (82) for $n=1$ and $m=0$, and get taking care that $\gamma_{1}=1$, the equation

$$
\mathbb{D}_{x}\left(\mathcal{L}_{1}\right)=\alpha \lambda_{1} P_{1} \mathcal{L}
$$

which is equivalent to

$$
\left\langle\psi \mathcal{L}, U_{2} \mathbb{D}_{x}^{2} F_{n}\right\rangle+\left\langle\phi \mathcal{L}, \mathbb{S}_{x} \mathbb{D}_{x} F_{n}\right\rangle-\left\langle\mathcal{L}, \alpha \lambda_{1} P_{1} F_{n}\right\rangle=0, \forall n \geq 0 .
$$


Next, we use the relations (52) and (53) for $f=F_{n}$

$$
\begin{aligned}
\mathbb{S}_{x} \mathbb{D}_{x} F_{n} & =\alpha \mathbb{D}_{x} \mathbb{S}_{x} F_{n}-\mathbb{D}_{x}\left(U_{1} \mathbb{D}_{x} F_{n}\right) \\
U_{2} \mathbb{D}_{x}^{2} F_{n} & =\alpha \mathbb{S}_{x}^{2} F_{n}-\mathbb{S}_{x}\left(U_{1} \mathbb{D}_{x} F_{n}\right)-\alpha F_{n},
\end{aligned}
$$

in Equation (91) to get

$$
\left\langle\mathbb{S}_{x}(\psi \mathcal{L})-\mathbb{D}_{x}(\phi \mathcal{L}), \alpha \mathbb{S}_{x} F_{n}-U_{1} \mathbb{D}_{x} F_{n}\right\rangle-\alpha\left\langle\mathcal{L},\left(\psi+\lambda_{1} P_{1}\right) F_{n}\right\rangle=0 .
$$

Because $\psi+\lambda_{1} P_{1}=0$, we then have:

$$
\left\langle\mathbb{S}_{x}(\psi \mathcal{L})-\mathbb{D}_{x}(\phi \mathcal{L}), \alpha \mathbb{S}_{x} F_{n}-U_{1} \mathbb{D}_{x} F_{n}\right\rangle=0 .
$$

Since $\alpha \mathbb{S}_{x} F_{n}-U_{1} \mathbb{D}_{x} F_{n}=\left(\alpha \alpha_{n}-\left(\alpha^{2}-1\right) \gamma_{n}\right) F_{n}+$ lower terms and $\alpha \alpha_{n}-\left(\alpha^{2}-1\right) \gamma_{n} \neq 0, \forall n \geq 0$, $\left(\alpha \mathbb{S}_{x} F_{n}-U_{1} \mathbb{D}_{x} F_{n}\right)_{n}$ forms a basis of $\mathbb{C}[x]$. We therefore deduce that

$$
\mathbb{S}_{x}(\psi \mathcal{L})-\mathbb{D}_{x}(\phi \mathcal{L})=0 .
$$

Step 3: $(a)+(b)+(c) \Rightarrow(f) \Rightarrow(a)$.

Step $3.1(a)+(b)+(c) \Rightarrow(f)$

We assume $(a)$ and therefore, $(b)$ and $(c)$. Expansion of the polynomial $\mathbb{S}_{x} Q_{n, m}$ in the basis $\left(Q_{j, m+1}\right)_{j \geq 0}$

$$
\mathbb{S}_{x} Q_{n, m}=\sum_{k=0}^{n} b_{n, k} Q_{k, m+1}
$$

yields

$$
b_{n, j}\left\langle\mathcal{L}_{m+1}, Q_{j, m+1}^{2}\right\rangle=\left\langle\mathcal{L}_{m+1},\left[\mathbb{S}_{x} Q_{n, m}\right] Q_{j, m+1}\right\rangle, 0 \leq j \leq n .
$$

First we use the second-order divided-difference Equation (79) for $Q_{j, m+1}, 1 \leq j \leq n$, next the product rules (19)-(20), then the Pearson equation for $\mathcal{L}_{m+1}$ (74) and finally Equation (68), and take into account the fact that thanks to Proposition $5, \lambda_{n, m} \neq 0$, for $n \geq 1$, to get

$$
\begin{aligned}
b_{n, j}\left\langle\mathcal{L}_{m+1}, Q_{j, m}^{2}\right\rangle= & \frac{-1}{\lambda_{j, m+1}}\left\langle\mathcal{L}_{m+1}, \mathbb{S}_{x} Q_{n, m}\left\{\phi^{(m+1)} \mathbb{D}_{x}^{2} Q_{j, m+1}+\psi^{(m+1)} \mathbb{S}_{x} \mathbb{D}_{x} Q_{j, m+1}\right\}\right\rangle \\
= & \frac{-1}{\lambda_{j, m+1}}\left\langle\phi^{(m+1)} \mathcal{L}_{m+1}, \mathbb{D}_{x}\left(Q_{n, m} \mathbb{D}_{x} Q_{j, m+1}\right)-\mathbb{D}_{x} Q_{n, m} \mathbb{S}_{x} \mathbb{D}_{x} Q_{j, m+1}\right\rangle \\
& +\frac{-1}{\lambda_{j, m+1}}\left\langle\psi^{(m+1)} \mathcal{L}_{m+1}, \mathbb{S}_{x}\left(Q_{n, m} \mathbb{D}_{x} Q_{j, m+1}\right)-U_{2} \mathbb{D}_{x} Q_{n, m} \mathbb{D}_{x}^{2} Q_{j, m+1}\right\rangle \\
= & \frac{\gamma_{n}}{\lambda_{j, m+1}}\left\langle\mathcal{L}_{m+1}, Q_{n-1, m+1}\left(\phi^{(m+1)} \mathbb{S}_{x} \mathbb{D}_{x} Q_{j, m+1}+U_{2} \psi^{(m+1)} \mathbb{D}_{x}^{2} Q_{j, m+1}\right)\right\rangle .
\end{aligned}
$$

Since $\phi^{(m+1)} \mathbb{S}_{x} \mathbb{D}_{x} Q_{j, m+1}+U_{2} \psi^{(m+1)} \mathbb{D}_{x}^{2} Q_{j, m+1}$ is of degree at most $j+1$, we use the orthogonality of $\left(Q_{n, m+1}\right)_{n}$ with respect to $\mathcal{L}_{m+1}$ to deduce that the previous expression vanishes for $j+1<n-1$. Therefore, $b_{n, j}=0,0<j<n-2$.

For $j=n-2$ with $n>2$, we get using the first line of the previous equation together with Property (59) for $\mathcal{L}=\mathcal{L}_{m+1}, \phi=\phi_{m+1}, \psi=\psi_{m+1}, f=Q_{n-2, m+1}$ and $g=\mathbb{S}_{x} Q_{n, m}$

$$
\begin{aligned}
b_{n, n-2}\left\langle\mathcal{L}_{m+1}, Q_{n-2, m}^{2}\right\rangle & =\frac{-1}{\lambda_{n-2, m+1}}\left\langle\mathcal{L}_{m+1}, \mathbb{S}_{x} Q_{n, m}\left\{\phi^{(m+1)} \mathbb{D}_{x}^{2} Q_{n-2, m+1}+\psi^{(m+1)} \mathbb{S}_{x} \mathbb{D}_{x} Q_{n-2, m+1}\right\}\right\rangle \\
& =\frac{-1}{\lambda_{n-2, m+1}}\left\langle\mathcal{L}_{m+1}, Q_{n-2, m+1}\left\{\phi^{(m+1)} \mathbb{D}_{x}^{2} \mathbb{S}_{x} Q_{n, m}+\psi^{(m+1)} \mathbb{S}_{x} \mathbb{D}_{x} \mathbb{S}_{x} Q_{n, m}\right\}\right\rangle \\
& =\frac{-\alpha_{n} \gamma_{n}\left(\gamma_{n-1} \phi_{2}^{(m)}+\alpha_{n-1} \psi_{1}^{(m)}\right)}{\lambda_{n-2, m+1}}\left\langle\mathcal{L}_{m+1}, Q_{n-2, m+1}^{2}\right\rangle .
\end{aligned}
$$


Therefore, we conclude by means of Proposition 5 with $\mathcal{L}$ replaced by $\mathcal{L}_{m+1}$, that $b_{n, n-2} \neq 0, n>2$.

For $j=0$, since $\lambda_{0, m}=0$, we cannot use the previous method. Instead, we use Relation (78) to transform (92) into

$$
\begin{aligned}
b_{n, 0}\left\langle\mathcal{L}_{m+1}, Q_{0, m+1}^{2}\right\rangle & =\left\langle\mathcal{L}_{m+1}, \mathbb{S}_{x} Q_{n, m}\right\rangle \\
& =\left\langle\mathbb{D}_{x}\left(U_{2} \psi^{(m)} \mathcal{L}_{m}\right)-\mathbb{S}_{x}\left(\phi^{(m)} \mathcal{L}_{m}\right), \mathbb{S}_{x} Q_{n, m}\right\rangle \\
& =-\left\langle U_{2} \psi^{(m)} \mathcal{L}_{m}, \mathbb{D}_{x} \mathbb{S}_{x} Q_{n, m}\right\rangle-\left\langle\phi^{(m)} \mathcal{L}_{m}, \mathbb{S}_{x}^{2} Q_{n, m}\right\rangle
\end{aligned}
$$

Next, we use Relations (52) and (53) for $f=Q_{n, m}$

$$
\begin{aligned}
\mathbb{D}_{x} \mathbb{S}_{x} Q_{n, m} & =\frac{1}{\alpha} \mathbb{D}_{x}\left(U_{1}(s) \mathbb{D}_{x} Q_{n, m}\right)+\frac{1}{\alpha} \mathbb{S}_{x} \mathbb{D}_{x} Q_{n, m} \\
\mathbb{S}_{x}^{2} Q_{n, m} & =\frac{1}{\alpha} \mathbb{S}_{x}\left(U_{1}(s) \mathbb{D}_{x} Q_{n, m}\right)+\frac{1}{\alpha} U_{2}(s) \mathbb{D}_{x}^{2} Q_{n, m}+Q_{n, m}
\end{aligned}
$$

and Relation (78) again to obtain

$$
\begin{aligned}
b_{n, 0}\left\langle\mathcal{L}_{m+1}, Q_{0, m+1}^{2}\right\rangle & =\frac{1}{\alpha}\left\langle\mathcal{L}_{m+1}, U_{1} \mathbb{D}_{x} Q_{n, m}\right\rangle \\
& -\frac{1}{\alpha}\left\langle\mathcal{L}_{m}, U_{2}\left(\phi^{(m)} \mathbb{D}_{x}^{2} Q_{n, m}+\psi^{(m)} \mathbb{S}_{x} \mathbb{D}_{x} Q_{n, m}\right)+\phi^{(m)} Q_{n, m}\right\rangle .
\end{aligned}
$$

Next we use Relation (68) and the Property (59) for $\phi=\phi^{(m)}, \psi=\psi^{(m)}, f=Q_{n, m}$ and $g=U_{2}$ to transform the previous equation into

$$
\begin{aligned}
b_{n, 0}\left\langle\mathcal{L}_{m+1}, Q_{0, m+1}^{2}\right\rangle & =\frac{\gamma_{n}}{\alpha}\left\langle\mathcal{L}_{m+1}, U_{1} Q_{n-1, m+1}\right\rangle \\
& -\frac{1}{\alpha}\left\langle\mathcal{L}_{m}, Q_{n, m}\left(\phi^{(m)} \mathbb{D}_{x}^{2} U_{2}+\psi^{(m)} \mathbb{S}_{x} \mathbb{D}_{x} U_{2}\right)\right\rangle+\frac{1}{\alpha}\left\langle\mathcal{L}_{m}, \phi^{(m)} Q_{n, m}\right\rangle .
\end{aligned}
$$

Therefore, $b_{n, 0}=0$ for $n>2$ thanks to the orthogonality of $\left(Q_{n, m}\right)_{n}$ and $\left(Q_{n, m+1}\right)_{n}$ with respect to $\mathcal{L}_{m}$ and $\mathcal{L}_{m+1}$ respectively. Hence,

$$
b_{n, j}=0,0 \leq j \leq n-3,
$$

and

$$
\begin{aligned}
\mathbb{S}_{x} Q_{n, m} & =b_{n, n} Q_{n, m+1}+b_{n, n-1} Q_{n-1, m+1}+b_{n, n-2} Q_{n-2, m+1}, \forall n \geq 1, \\
& =\frac{b_{n, n}}{\gamma_{n+1}} \mathbb{D}_{x} Q_{n+1, m}+\frac{b_{n, n-1}}{\gamma_{n}} \mathbb{D}_{x} Q_{n, m}+\frac{b_{n, n-2}}{\gamma_{n-1}} \mathbb{D}_{x} Q_{n-1, m}, \forall n \geq 1, \\
& =b_{n, n+1}^{m} \mathbb{D}_{x} Q_{n+1, m}+b_{n, n}^{m} \mathbb{D}_{x} Q_{n, m}+b_{n, n-1}^{m} \mathbb{D}_{x} Q_{n-1, m}, \forall n \geq 1,
\end{aligned}
$$

where

$$
b_{n, n+j}^{m}=\frac{b_{n, n+j-1}}{\gamma_{n+j}},-1 \leq j \leq 1
$$

with

$$
b_{n, n-1}^{m}=\frac{b_{n, n-2}}{\gamma_{n-1}} \neq 0, n>2 .
$$

Step 3.2 $(f) \Rightarrow(a)$

We assume Property $(f)$, and denote by $\left(\hat{Q}_{n, m}\right)_{n}$ the dual basis associated to $\left(Q_{n, m}\right)_{n}$. Then, expansion of the linear functional $\alpha \mathbb{S}_{x} \hat{Q}_{0,1}-\mathbb{D}_{x}\left(U_{1} \hat{Q_{0,1}}\right)$ in the dual basis $\left(\hat{Q}_{n, 0}\right)_{n}=\left(\hat{P}_{n}\right)_{n}$ of $\left(P_{n}\right)_{n}$

$$
\alpha \mathbb{S}_{x} \hat{Q}_{0,1}-\mathbb{D}_{x}\left(U_{1} \hat{Q}_{0,1}\right)=\sum_{k \geq 0} c_{k} \hat{P}_{k}
$$


yields after using (28)

$$
c_{j}=\left\langle\alpha \mathbb{S}_{x} \hat{Q}_{0,1}-\mathbb{D}_{x}\left(U_{1} \hat{Q}_{0,1}\right), P_{j}\right\rangle=\left\langle\hat{Q}_{0,1}, \alpha \mathbb{S}_{x} P_{j}+U_{1} \mathbb{D}_{x} P_{j}\right\rangle .
$$

Application of $\mathbb{D}_{x}$ on both sides of the recurrence relation for the orthogonal family $\left(P_{n}\right)_{n}$

$$
x(s) P_{j}=c_{j, j+1} P_{j+1}+c_{j, j} P_{j}+c_{j, j-1} P_{j-1},
$$

and use of the product rule (19) give

$$
\alpha x(s) \mathbb{D}_{x} P_{j}+\mathbb{S}_{x} P_{j}=c_{j, j+1} \mathbb{D}_{x} P_{j+1}+c_{j, j} \mathbb{D}_{x} P_{j}+c_{j, j-1} \mathbb{D}_{x} P_{j-1}
$$

Then use of Relation (28), the previous equation as well as the structure relation (85) for $m=0$ and $n=j$ produces the relation

$$
U_{1} \mathbb{D}_{x} P_{j}=d_{j, j+1} \mathbb{D}_{x} P_{j+1}+d_{j, j} \mathbb{D}_{x} P_{j}+d_{j, j-1} \mathbb{D}_{x} P_{j-1},
$$

which combined with the structure relation (85) for $m=0$ and $n=j$ gives

$$
\alpha \mathbb{S}_{x} P_{j}+U_{1} \mathbb{D}_{x} P_{j}=e_{j, j+1} \mathbb{D}_{x} P_{j+1}+e_{j, j} \mathbb{D}_{x} P_{j}+e_{j, j-1} \mathbb{D}_{x} P_{j-1}
$$

Finally we deduce from (94), the previous relation and (67) for $m=1$

$$
\begin{aligned}
c_{j}=\left\langle\hat{Q}_{0,1}, \alpha \mathbb{S}_{x} P_{j}+U_{1} \mathbb{D}_{x} P_{j}\right\rangle & =\left\langle\hat{Q}_{0,1}, e_{j, j+1} \mathbb{D}_{x} P_{j+1}+e_{j, j} \mathbb{D}_{x} P_{j}+e_{j, j-1} \mathbb{D}_{x} P_{j-1}\right\rangle \\
& =\left\langle-\mathbb{D}_{x} \hat{Q}_{0,1}, e_{j, j+1} P_{j+1}+e_{j, j} P_{j}+e_{j, j-1} P_{j-1}\right\rangle \\
& =\left\langle\gamma_{1} \hat{Q}_{1,0}, e_{j, j+1} P_{j+1}+e_{j, j} P_{j}+e_{j, j-1} P_{j-1}\right\rangle \\
& =\gamma_{1}\left\langle\hat{P}_{1}, e_{j, j+1} P_{j+1}+e_{j, j} P_{j}+e_{j, j-1} P_{j-1}\right\rangle \\
& =0 \text { for } j-1>1 .
\end{aligned}
$$

Therefore

$$
\begin{aligned}
\alpha \mathbb{S}_{x} \hat{Q}_{0,1}-\mathbb{D}_{x}\left(U_{1} \hat{Q}_{0,1}\right) & =c_{0} \hat{P}_{0}+c_{1} \hat{P}_{1}+c_{2} \hat{P}_{2} \\
& =\phi \mathcal{L}
\end{aligned}
$$

where

$$
\phi=\frac{c_{0} P_{0}}{\left\langle\mathcal{L}, P_{0} P_{0}\right\rangle}+\frac{c_{1} P_{1}}{\left\langle\mathcal{L}, P_{1} P_{1}\right\rangle}+\frac{c_{2} P_{2}}{\left\langle\mathcal{L}, P_{2} P_{2}\right\rangle},
$$

thanks to (66). Application of the linear functional $\mathbb{D}_{x}\left(\alpha \mathbb{S}_{x} \hat{Q}_{0,1}-\mathbb{D}_{x}\left(U_{1} \hat{Q}_{0,1}\right)\right)$ to the polynomial $P_{n}$, and use of Relations (26) and (67) yields

$$
\begin{aligned}
\mathbb{D}_{x}(\phi \mathcal{L})=\left\langle\mathbb{D}_{x}\left(\alpha \mathbb{S}_{x} \hat{Q}_{0,1}-\mathbb{D}_{x}\left(U_{1} \hat{Q}_{0,1}\right)\right), P_{n}\right\rangle & =-\left\langle\hat{Q}_{0,1}, \alpha \mathbb{S}_{x} \mathbb{D}_{x} P_{n}+U_{1} \mathbb{D}_{x}^{2} P_{n}\right\rangle \\
& =-\left\langle\hat{Q}_{0,1}, \mathbb{D}_{x} \mathbb{S}_{x} P_{n}\right\rangle \\
& =\left\langle\mathbb{D}_{x} \hat{Q}_{0,1}, \mathbb{S}_{x} P_{n}\right\rangle \\
& =-\gamma_{1}\left\langle\hat{Q}_{1,0}, \mathbb{S}_{x} P_{n}\right\rangle \\
& =-\gamma_{1}\left\langle\mathbb{S}_{x} \hat{P}_{1}, P_{n}\right\rangle \\
& =\left\langle\mathbb{S}_{x}(\psi \mathcal{L}), P_{n}\right\rangle
\end{aligned}
$$

where $\psi=-\gamma_{1} \frac{P_{1}}{\left\langle\mathcal{L}, P_{1} P_{1}\right\rangle}$. Summing up, we have

$$
\mathbb{D}_{x}(\phi \mathcal{L})=\mathbb{S}_{x}(\psi \mathcal{L})
$$

where $\phi$ is a polynomial of degree at most two and $\psi$ a first-degree polynomial.

Step 4: $(c)+(f) \Rightarrow(e) \Rightarrow(a)$. 
Step $4.1(c)+(f) \Rightarrow(e)$

We assume Property $(f)$ and make use of Property $(c)$ since we have proved above that $(f) \Longleftrightarrow$ $(a) \Longleftrightarrow(b) \Longleftrightarrow(c)$.

Application of $\mathbb{D}_{x}$ to both sides of (85) followed by the multiplication by $\phi^{(m)}$ and use of (79) gives for $n \geq 1$

$$
\begin{aligned}
\phi^{(m)} \mathbb{D}_{x} \mathbb{S}_{x} Q_{n, m}= & b_{n, n+1}^{m} \phi^{(m)} \mathbb{D}_{x}^{2} Q_{n+1, m}+b_{n, n}^{m} \phi^{(m)} \mathbb{D}_{x}^{2} Q_{n, m}+b_{n, n-1}^{m} \phi^{(m)} \mathbb{D}_{x}^{2} Q_{n-1, m} \\
= & -b_{n, n+1}^{m}\left(\psi^{(m)} \mathbb{S}_{x} \mathbb{D}_{x} Q_{n+1, m}+\lambda_{n+1, m} Q_{n+1, m}\right) \\
& -b_{n, n}^{m}\left(\psi^{(m)} \mathbb{S}_{x} \mathbb{D}_{x} Q_{n, m}+\lambda_{n, m} Q_{n, m}\right) \\
& -b_{n, n-1}^{m}\left(\psi^{(m)} \mathbb{S}_{x} \mathbb{D}_{x} Q_{n-1, m}+\lambda_{n-1, m} Q_{n-1, m}\right) \\
= & -\psi^{(m)} \mathbb{S}_{x}\left[b_{n, n+1}^{m} \mathbb{D}_{x} Q_{n+1, m}+b_{n, n}^{m} \mathbb{D}_{x} Q_{n, m}+b_{n, n-1}^{m} \mathbb{D}_{x} Q_{n-1, m}\right] \\
& -b_{n, n+1}^{m} \lambda_{n+1, m} Q_{n+1, m}-b_{n, n}^{m} \lambda_{n, m} Q_{n, m}-b_{n, n-1}^{m} \lambda_{n-1, m} Q_{n-1, m}
\end{aligned}
$$

A second use of (85) transforms the previous equation into

$$
\phi^{(m)} \mathbb{D}_{x} \mathbb{S}_{x} Q_{n, m}+\psi^{(m)} \mathbb{S}_{x} Q_{n, m}=-b_{n, n+1}^{m} \lambda_{n+1, m} Q_{n+1, m}-b_{n, n}^{m} \lambda_{n, m} Q_{n, m}-b_{n, n-1}^{m} \lambda_{n-1, m} Q_{n-1, m}
$$

Therefore,

$$
\phi^{(m)} \mathbb{D}_{x} \mathbb{S}_{x} Q_{n, m}+\psi^{(m)} \mathbb{S}_{x}^{2} Q_{n, m}=a_{n, n+1}^{m} Q_{n+1, m}+a_{n, n}^{m} Q_{n, m}+a_{n, n-1}^{m} Q_{n-1, m}, n \geq 1,
$$

with

$$
a_{n, n+j}^{m}=-b_{n, n+j}^{m} \lambda_{n+j, m},-1 \leq j \leq 1 .
$$

In addition, $a_{n, n-1}^{m}=-b_{n, n-1}^{m} \lambda_{n-1, m} \neq 0$ for $n>2$ since $b_{n, n-1}^{m} \neq 0$ and $\lambda_{n-1, m} \neq 0$ both for $n>2$.

Step $4.2(e) \Rightarrow(a)$

We assume $(e)$ and obtain using (85)

$$
\begin{aligned}
\left\langle\mathbb{D}_{x}(\phi \mathcal{L})-\mathbb{S}_{x}(\psi \mathcal{L}), \mathbb{S}_{x} Q_{n, 0}\right\rangle & =-\left\langle\mathcal{L}, \phi \mathbb{D}_{x} \mathbb{S}_{x} Q_{n, 0}+\psi \mathbb{S}_{x}^{2} Q_{n, 0}\right\rangle \\
& =-\left\langle\mathcal{L}, a_{n, n+1}^{0} Q_{n+1,0}+a_{n, n}^{0} Q_{n, 0}+a_{n, n-1}^{0} Q_{n-1,0}\right\rangle \\
& =-\left\langle\mathcal{L}, a_{n, n+1}^{0} P_{n+1}+a_{n, n}^{0} P_{n}+a_{n, n-1}^{0} P_{n-1}\right\rangle \\
& =0, \text { for } n \geq 2
\end{aligned}
$$

For $n=1$ and for $n=0$, we have

$$
\begin{aligned}
\phi \mathbb{D}_{x} \mathbb{S}_{x} P_{1}+\psi \mathbb{S}_{x}^{2} P_{1} & =a_{1,2}^{0} P_{2}+a_{1,1}^{0} P_{1}+a_{1,0}^{0} P_{0}=a_{1,2}^{0} P_{2}+a_{1,1}^{0} P_{1}, \\
\phi \mathbb{D}_{x} \mathbb{S}_{x} P_{0}+\psi \mathbb{S}_{x}^{2} P_{0} & =\psi=a_{0,1}^{0} P_{1}+a_{0,0}^{0} P_{0}=a_{0,1}^{0} P_{1},
\end{aligned}
$$

since $\lambda_{0, m}=0$,

$$
a_{1,0}^{0}=-b_{1,0}^{0} \lambda_{0,0}=0 \text {, and } a_{0,0}^{0}=-b_{0,0}^{0} \lambda_{0,0}=0 .
$$

Summing up, we have

$$
\left\langle\mathbb{D}_{x}(\phi \mathcal{L})-\mathbb{S}_{x}(\psi \mathcal{L}), \mathbb{S}_{x} Q_{n, 0}\right\rangle=0, n \geq 0, \text { and } \mathbb{D}_{x}(\phi \mathcal{L})=\mathbb{S}_{x}(\psi \mathcal{L})
$$

Step $5(a) \Leftrightarrow(g)$

Step $5.1(a) \Rightarrow(g)$

Assuming $(a)$, we take the formal Stieltjes function of both sides of the Pearson equation (73) to get

$$
S\left[\mathbb{D}_{x}(\phi \mathcal{L})\right](x(s))=S\left[\mathbb{S}_{x}(\psi \mathcal{L})\right](x(s)) .
$$


Use of (39), (40) and (42) transforms the previous equation into

$$
\begin{aligned}
\mathbb{D}_{x}[S(\phi \mathcal{L})(x(s))] & =\alpha \mathbb{S}_{x}[S(\psi \mathcal{L})(x(s))]+U_{1}(x(s)) \mathbb{D}_{x}[S(\psi \mathcal{L})(x(s))] \\
& \mathbb{1} \\
\mathbb{D}_{x}\left[\phi(x(s)) S(\mathcal{L})(x(s))+\left(\mathcal{L} \theta_{0} \phi\right)(x(s))\right] & =\alpha \mathbb{S}_{x}\left[\psi(x(s)) S(\mathcal{L})(x(s))+\left(\mathcal{L} \theta_{0} \psi\right)(x(s))\right] \\
& +U_{1}(x(s)) \mathbb{D}_{x}\left[\psi(x(s)) S(\mathcal{L})(x(s))+\left(\mathcal{L} \theta_{0} \psi\right)(x(s))\right] .
\end{aligned}
$$

Finally, we use the product rules (19), (20) and the definition of $\mathcal{L} \theta_{0} f$ given by (43) to obtain the following Riccati equation for $S(\mathcal{L})$

$$
A(x(s)) \mathbb{D}_{x} S(\mathcal{L})(x(s))=B(x(s)) \mathbb{S}_{x} S(\mathcal{L})(x(s))+C(x(s))
$$

where

$$
\begin{aligned}
A & =\mathbb{S}_{x} \phi-\alpha \psi_{1} U_{2}-U_{1} \mathbb{S}_{x} \psi \\
B & =\alpha \mathbb{S}_{x} \psi+\psi_{1} U_{1}-\mathbb{D}_{x} \phi \\
C & =\left(\alpha \psi_{1}-\phi_{2}\right)\langle\mathcal{L}, 1\rangle
\end{aligned}
$$

where $\phi_{2}$ and $\psi_{1}$ are given by (81).

Step $5.2(g) \Rightarrow(a)$

Assuming (a), we use Equations (39) and (40) to transform the Riccati Equation (86) into

$$
\left(A(x)+\frac{U_{1}}{\alpha} B(x)\right) S\left(\mathbb{D}_{x} \mathcal{L}\right)=\frac{1}{\alpha} B(x) S\left(\mathbb{S}_{x} \mathcal{L}\right)+C(x) .
$$

By means of (42), the latter equation is equivalent to

$$
S\left[\left(A(x)+\frac{U_{1}}{\alpha} B(x)\right) \mathbb{D}_{x} \mathcal{L}-\frac{1}{\alpha} B(x) \mathbb{S}_{x} \mathcal{L}\right]=C(x)-\frac{1}{\alpha}\left(\mathbb{S}_{x} \mathcal{L}\right) \theta_{0} B(x(s))+\left(\mathbb{D}_{x} \mathcal{L}\right) \theta_{0}\left(A(x)+\frac{U_{1}}{\alpha} B(x)\right) .
$$

The right-hand side of the previous relation is a polynomial while the left-hand side is, by definition of the Stieltjes function of a given linear functional given by (38), an infinite linear combination of $\left\{\frac{1}{F_{n+1}}, n \in \mathbb{N}\right\}$. Therefore, both sides of the previous equation vanish and we obtain:

$$
\left(A(x)+\frac{U_{1}}{\alpha} B(x)\right) \mathbb{D}_{x} \mathcal{L}-\frac{1}{\alpha} B(x) \mathbb{S}_{x} \mathcal{L}=0
$$

and

$$
C(x(s))=\frac{1}{\alpha}\left(\mathbb{S}_{x} \mathcal{L}\right) \theta_{0} B(x(s))-\left(\mathbb{D}_{x} \mathcal{L}\right) \theta_{0}\left(A(x)+\frac{U_{1}}{\alpha} B(x)\right) .
$$

Using Relations (62) and (63), Relation (96) becomes

$$
\mathbb{D}_{x}\left[\left(\mathbb{S}_{x} H(x)+U_{2} \mathbb{D}_{x} K(x)\right) \mathcal{L}\right]-\mathbb{S}_{x}\left[\left(\mathbb{D}_{x} H(x)+\mathbb{S}_{x} K(x)\right) \mathcal{L}\right]=0,
$$

where

$$
H(x)=A(x)+\frac{U_{1}}{\alpha} B(x), \quad K(x)=\frac{1}{\alpha} B(x) .
$$

Since $A$ and $B$ are polynomials of degree at most two and one respectively, the polynomials

$$
\phi=\mathbb{S}_{x}\left(A(x)+\frac{U_{1}}{\alpha} B(x)\right)-\frac{U_{2}}{\alpha} \mathbb{D}_{x}(B(x)), \psi=\mathbb{D}_{x}\left(A(x)+\frac{U_{1}}{\alpha} B(x)\right)-\frac{1}{\alpha} \mathbb{S}_{x}(B(x))
$$

are of degree at most two and one respectively. Next, we write $\psi=u P_{1}+v$ and obtain $v\langle\mathcal{L}, 1\rangle=\langle\mathcal{L}, \psi\rangle$. Application of both sides of the Pearson equation (97) to the constant polynomial 1 yields $\langle\mathcal{L}, \psi\rangle=0$. Therefore, $\psi=u P_{1}$ is of degree exactly 1 . 


\section{Important Connections}

\subsection{Connection with the Structure Relation by Koornwinder}

The structure relation (14) given by Koornwinder [14] is related to our results in the following way:

Theorem 5 The structure relation (14) for classical orthogonal polynomials $\left(P_{n}\right)_{n}$ on a non-uniform lattice satisfying (17) can be expressed in terms of the operator $\mathbb{D}_{x}$ and $\mathbb{S}_{x}$ as

$$
\mathbb{L}\left(p_{n}\right)(x(s))=\zeta\left(2 \psi \mathbb{S}_{x}^{2}+2 \phi \mathbb{D}_{x} \mathbb{S}_{x}-\psi \mathbb{I}\right) p_{n}(x(s))=\gamma_{n} A_{n} p_{n+1}(x)-\gamma_{n-1} C_{n} p_{n-1}(x),
$$

where $\zeta$ is a constant term.

For the specific case of the Askey-Wilson polynomials, the coefficients $\phi$ and $\psi$ are given by [8]

$$
\begin{aligned}
& \phi(x(s))= 2(d c b a+1) x^{2}(s)-(a+b+c+d+a b c+a b d+a c d+b c d) x(s) \\
&+a b+a c+a d+b c+b d+c d-a b c d-1 \\
& \psi(x(s))= \frac{4(a b c d-1) q^{\frac{1}{2}} x(s)}{q-1}+\frac{2(a+b+c+d-a b c-a b d-a c d-b c d) q^{\frac{1}{2}}}{q-1} .
\end{aligned}
$$

Proof: We assume that $\left(p_{n}\right)_{n}$ is a family of polynomial orthogonal with respect to the linear functional $\mathcal{L}$ satisfying the Pearson equation

$$
\mathbb{D}_{x}(\phi \mathcal{L})=\mathbb{S}_{x}(\psi \mathcal{L})
$$

where $\phi$ is a polynomial of degree at most two and $\psi$ a first-degree polynomial. Because of the property (59), the operator $\mathbb{O}=\phi \mathbb{D}_{x}^{2}+\psi \mathbb{S}_{x} \mathbb{D}_{x}$ is symmetric with respect to the inner product

$$
(p, q)=\langle\mathcal{L}, p q\rangle, p, q \in \mathbb{R}[x(s)]
$$

that is,

$$
(\mathbb{O}(p), q)=(p, \mathbb{O}(q)), \forall p, q \in \mathbb{R}[x(s)] .
$$

Since $\left(\mathbb{O}\right.$ satisfies in addition the property $\mathbb{O}\left(p_{n}\right)=\lambda_{n} p_{n}$, with $\lambda_{n} \neq \lambda_{n-1}$, we deduce thanks to Proposition 2.2 of [14] that the commutator $\tilde{\mathbb{L}}$ defined by

$$
\tilde{\mathbb{L}}(p)(x(s))=[\mathbb{O}, X](x(s))=\mathbb{O}[x(s) p(x(s))]-x(s) \mathbb{O}(p)(x(s))
$$

is skew symmetric with respect to the inner product (100) and satisfies the structure relation (14). Computation using the product rules (19), (20), (26) and (27) give

$$
\tilde{\mathbb{L}}(p)(x(s))=\left(2 \psi \mathbb{S}_{x}^{2}+2 \phi \mathbb{D}_{x} \mathbb{S}_{x}-\psi \mathbb{I}\right) p_{n}(x(s))=\mathbb{L}(p)(x(s)) .
$$

For the recurrence relation (15) which is the specific case of the Askey-Wilson polynomials, in the first step, we deduce from the notation

$$
[z]=\frac{z+z^{-1}}{2}=\frac{q^{s}+q^{-s}}{2}=x(s)
$$

that

$$
\begin{aligned}
{[q z] } & =x(s+1),\left[\frac{z}{q}\right]=x(s-1) \\
x\left(s+\frac{1}{2}\right) & =[\sqrt{q} z]=\frac{\sqrt{q} z}{2}+\frac{1}{2 \sqrt{q} z},\left[\frac{z}{\sqrt{q}}\right]=x\left(s-\frac{1}{2}\right) .
\end{aligned}
$$

In the second step, we solve the linear equations

$$
\mathbb{D}_{x} \mathbb{S}_{x} f(x(s))=\frac{f(x(s+1))-f(x(s-1))}{x\left(s+\frac{1}{2}\right)-x\left(s-\frac{1}{2}\right)}, \mathbb{S}_{x}^{2} f(x(s))=\frac{f(x(s+1))+2 f(x(s))+f(x(s-1))}{4}
$$


to get

$$
\begin{aligned}
& f(x(s+1))=2 \mathbb{S}_{x}^{2} f(x(s))-f(x(s))+\left(x\left(s+\frac{1}{2}\right)-x\left(s-\frac{1}{2}\right)\right) \mathbb{D}_{x} \mathbb{S}_{x} f(x(s)), \\
& f(x(s-1))=2 \mathbb{S}_{x}^{2} f(x(s))-f(x(s))-\left(x\left(s+\frac{1}{2}\right)-x\left(s-\frac{1}{2}\right)\right) \mathbb{D}_{x} \mathbb{S}_{x} f(x(s)) .
\end{aligned}
$$

In the third step, we substitute (102) in the right-hand side of (15) to obtain an equation in which we substitute (104) and (105), then (103) to get an equation of the form

$$
\begin{aligned}
\mathbb{L}\left(p_{n}\right) & =\frac{q-1}{2 \sqrt{q}}\left(2 \psi([z]) \mathbb{S}_{x}^{2} p_{n}(x(s))+2 \phi([z]) \mathbb{D}_{x} \mathbb{S}_{x} p_{n}(x(s))-\psi([z]) p_{n}(x(s))\right) \\
& =\frac{q-1}{2 \sqrt{q}}\left(2 \psi(x(s)) \mathbb{S}_{x}^{2}+2 \phi(x(s)) \mathbb{D}_{x} \mathbb{S}_{x}-\psi(x(s)) \mathbb{I}\right) p_{n}(x(s))
\end{aligned}
$$

where $\phi$ and $\psi$ are those of the Askey-Wilson polynomials given above which appeared already in [8].

\subsection{Connection with some pioneering work by Magnus}

In the papers $[15,16]$, Magnus defined the Laguerre-Hahn orthogonal polynomials on the non-uniform lattice as the ones for which the formal Stieltjes series of the corresponding functional given by (37) satisfies a Riccati difference equation (see Equation (2.4) of [15]). He also proved that for a non-uniform lattice, the associated Laguerre-Hahn orthogonal polynomials are again Laguerre-Hahn orthogonal polynomials, and he recovered the associated Askey-Wilson polynomials as special case of the Laguerre-Hahn orthogonal polynomials.

The present work provides a bridge between the theory of Magnus based mainly on the Riccati equation satisfied by the formal Stieltjes function (37), and the theory of classical orthogonal polynomials based on the functional approach (which is already extended to the functional approach of the theory of semi-classical and Laguerre-Hahn orthogonal polynomials [10, 11]).

\section{Conclusion and Perspectives}

In this work, we have:

1. stated the Pearson-type equation for the linear functional of the corresponding classical orthogonal polynomials;

2. proved that the Pearson equation for the weight implies the one of the linear functional;

3. stated and proved using the functional approach seven equivalent characterization properties for classical orthogonal polynomials: the four properties given by Costas-Santos and Marcellán [5] but using the Pearson equation for the corresponding weight function, plus, the Pearson equation for the linear functional, the Rodrigues formula for the linear functional, the first structure relation and the Riccati equation for the formal Stieltjes function;

4. found the link between the structure relation given above by Koornwinder [14] and our second structure relation;

5. connected this work with the pioneering one by Magnus $[15,16]$, done using mainly the Riccati equation for the corresponding orthogonal family. 
Since the operator $\mathbb{D}_{x}$ reduces to the forward operator $\Delta$ and the Hahn operator $D_{q}\left(D_{q} f(s)=\frac{f(q s)-f(s)}{(q-1) s}\right)$ for the lattices $x(s)=s$ and $x(s)=q^{s}$ respectively [8], this work generalizes previous ones characterizing classical orthogonal polynomials by means of the above mentioned seven equivalent properties. Among these, we would like to mention $[1,2,17]$ for COP of a continuous variable, [12] for COP of a discrete variable, $[21,20,3]$ for COP of a $q$-discrete variable and $[5,14]$ for COP on a non-uniform lattice.

We end by mentioning that our work completes and generalizes the one of [5], connecting to the pioneering work done by Magnus. In addition, it has interesting perspective which is the completion and generalization of the work of Magnus $[15,16]$ by stating and proving - using the functional approach-the characterization theorems for the semi-classical and Laguerre-Hahn orthogonal polynomials on non-uniform lattices $[10,11])$. This will allow the study of the properties of new orthogonal polynomials obtained by modifications of the initial ones (see [6] and references therein).

\section{Acknowledgement}

The authors would like to acknowledge the various financial supports from the Alexander von Humboldt Foundation (Bonn, Germany), and particularly the Research Group Linkage between the University of Kassel and the University of Yaounde I (2009-2012) which enabled their stay in Germany (June-September 2009) and their participation to the $10^{\text {th }}$ Conference on Orthogonal Polynomials, Special Functions and Applications (Leuven, July 20-25, 2009) where part of this work was presented. Their sincere thanks go to Professor Wolfram Koepf for his kind hospitality.

In addition, the first author would also like to thank Professors David Bekolle (University of Ngaoundere, Cameroon) and Hjalmar Rosengren (Chalmers University of Technology, Göteborg, Sweden); the first for providing the funding via the International Mathematical Science Programme-IMSP (Upsala University, Sweden) for visit in Göteborg in August 2007 during which part of this work was initiated, and the second for his kind hospitality.

\section{References}

[1] W.A. Al-Salam, Characterization theorems for orthogonal polynomials, in: P. Nevai (Ed.), Orthogonal Polynomials: Theory and Practice, NATO ASI Series C, Vol. 294, Kluwer Academic Publishers, Dordrecht, 1990, 1-24.

[2] W.A. Al-Salam, T.S. Chihara, Another characterization of the classical orthogonal polynomials, SIAM J. Math. Anal. 3 (1972), 65-70.

[3] R. A. Alvarez-Nodarse, J. Arvesú, On the q-polynomials in the exponential lattice $x(s)=c_{1} q^{s}+c_{3}$, Integral Transform. Special Funct. 8 (1999), 299-324.

[4] N. M. Atakishiyev, M. Rahman and S. K. Suslov: On Classical Orthogonal Polynomials. Constructive Approximation. 11 (1995), 181-226.

[5] R. S. Costas-Santos, F. Marcellán, $q$-Classical Orthogonal Polynomials: A General Difference Calculuc Approach, Acta Appl. Math., published online on July 04, 2009.

[6] M. Foupouagnigni, W. Koepf and A. Ronveaux: Factorization of fourth-order differential equations for perturbed classical orthogonal polynomials, J. Comput. Appl. Math. 162 (2004), 299-326.

[7] M. Foupouagnigni, On Difference and Differential Equations for Modifications of Classical Orthogonal Polynomials, Habilitation thesis, University of Kassel, Kassel, Germany, 2006.

[8] M. Foupouagnigni, On difference equations for orthogonal polynomials on non-uniform lattices, J. Diff. Eqn. Appl, 14/2 (2008), 127-174. 
[9] M. Foupouagnigni, W. Koepf, M. Kenfack-Nangho and S. Mboutngam, On Solutions of Holonomic Divided-Difference Equations on non-uniform Lattices, Submitted. Accessible at: https://kobra.bibliothek.uni-kassel. de/handle/urn:nbn: de: hebis: $34-2010082534270$

[10] M. Foupouagnigni and S. Mboutngam, On semi-classical orthogonal polynomials on non-uniform lattices, In progress.

[11] M. Foupouagnigni and M. Kenfack-Nangho, Characterization theorem for Laguerre-Hahn orthogonal polynomials on non-uniform Lattices, In progress.

[12] A.G. García, F. Marcellán, L. Salto, A distributional study of discrete classical orthogonal polynomials, J. Comput. Appl. Math. 57 (1995), 147-162.

[13] R. Koekoek and R. Swarttouw: The Askey-scheme of hypergeometric orthogonal polynomials and its $q$-analogue. Report no. 98-17, (1998), Faculty of Information Technology and Systems, Delft University of Technology.

[14] T.H. Koornwinder, The structure relation for Askey-Wilson polynomials, J. Comput. Appl. Math. 207/2 (2007), 214-226.

[15] Magnus, A. P.: Associated Askey-Wilson polynomials as Laguerre-Hahn orthogonal polynomials. Lect. Notes in Math. 1329, 261-278. Springer, Berlin (1988)

[16] Magnus, A. P.: Special nonuniform lattice (snul) orthogonal polynomials on a discrete dense sets of points. J. Comput. Appl. Math. 65, 253-265 (1995)

[17] F. Marcellán, A. Branquinho, J. Petronilho, Classical orthogonal polynomials: a functional approach, Acta Appl. Math. 34 (1994), 203-283.

[18] P. Maroni. Le calcul des formes linéaires et les polynômes orthogonaux semi-classiques, in M. Alfaro et al., (Eds) "Orthogonal Polynomials and their Applications", Lecture Notes in Mathematics, 1329 Springer-Verlag, Berlin, 1986, 279-290.

[19] P. Maroni, Une théorie algébrique des polynômes orthogonaux. Application aux polynômes orthogonaux semi-classiques. In: C. Brezinski, L. Gori and A. Ronveaux, (Eds), Orthogonal Polynomials and their Applications, IMACS Annals on Computing and Applied Mathematics Vol. 9, J.C. Baltzer AG Publishers (1991), pp. 95-130.

[20] J.C. Medem, F. Marcellán, q-Classical polynomials: a very classical approach, Electron. Trans. Numer. Annal. 9 (1999), 112-127.

[21] J. C. Medem, R. Álvarez-Nodarse, and F. Marcellán, On the $q$-polynomials: a distributional study , J. Comput. Appl. Math. 135 (2001), 157-196

[22] M. B. Monagan, K. O. Geddes, K. M. Heal, G. Labahn, S. M. Vorkoetter, J. McCarron and P. DeMarco: Maple 9 Introductory Programming Guide, Maplesoft, 2003.

[23] T. Sprenger, Algorithmen für $q$-holonome Funktionen und $q$-hypergeometrische Reihen, PhD thesis. Universität Kassel, 2009. Available at: urn:nbn:de:hebis:34-2009072129130.

[24] S. K. Suslov: The theory of difference analogues of special functions of hypergeometric type. Russian Math, Surveys. 44 (1989), 227-278.

[25] S. K. Suslov, An Introduction to Basic Fourier Series, Kluwer Academic Publishers, Dordrecht/Boston/London, 2003. 